\title{
On equivalence relations second order definable over $H(\kappa)$
}

\author{
by \\ Saharon Shelah (Jerusalem and New Brunswick, NJ) and \\ Pauli Väisänen (Helsinki)
}

\begin{abstract}
Let $\kappa$ be an uncountable regular cardinal. Call an equivalence relation on functions from $\kappa$ into 2 second order definable over $H(\kappa)$ if there exists a second order sentence $\phi$ and a parameter $P \subseteq H(\kappa)$ such that functions $f$ and $g$ from $\kappa$ into 2 are equivalent iff the structure $\langle H(\kappa), \in, P, f, g\rangle$ satisfies $\phi$. The possible numbers of equivalence classes of second order definable equivalence relations include all the nonzero cardinals at most $\kappa^{+}$. Additionally, the possibilities are closed under unions and products of at most $\kappa$ cardinals. We prove that these are the only restrictions: Assuming that GCH holds and $\lambda$ is a cardinal with $\lambda^{\kappa}=\lambda$, there exists a generic extension where all the cardinals are preserved, there are no new subsets of cardinality $<\kappa, 2^{\kappa}=\lambda$, and for all cardinals $\mu$, the number of equivalence classes of some second order definable equivalence relation on functions from $\kappa$ into 2 is $\mu$ iff $\mu$ is in $\Omega$, where $\Omega$ is any prearranged subset of $\lambda$ such that $0 \notin \Omega, \Omega$ contains all the nonzero cardinals $\leq \kappa^{+}$, and $\Omega$ is closed under unions and products of at most $\kappa$ cardinals.
\end{abstract}

1. Introduction. We deal with equivalence relations which are second order definable over $H(\kappa)$, where $\kappa$ is an uncountable regular cardinal. We show that it is possible to have a generic extension where the numbers of equivalence classes of such equivalence relations are in a prearranged set. This is applied to the problem of the possible numbers of strongly equivalent nonisomorphic models of weakly compact cardinality in [SV]. Namely, for a weakly compact cardinal $\kappa$, there exists a model of cardinality $\kappa$ with $\mu$ strongly equivalent nonisomorphic models if, and only if, there exists an equivalence relation which is $\Sigma_{1}^{1}$-definable over $H(\kappa)$ and it has $\mu$ equivalence classes (for an explanation of $\Sigma_{1}^{1}$ see Definition 3.1). The paper [SV]

2000 Mathematics Subject Classification: Primary 03E35; Secondary 03C55, 03C75.

Key words and phrases: second order definable equivalence relations, number of models, infinitary logic.

Research of S. Shelah supported by the United States-Israel Binational Science Foundation. Publication 719.

Research of P. Väisänen partially supported by Academy of Finland grant 40734. 
can be read independently of this paper, if the reader accepts the present conclusion on faith. For a history and other applications of this type of equivalence relations see [Sheb, Shea]. In the first paper Shelah mostly tries to get results in the other direction than in the present paper, i.e., that the possible number of equivalence classes is limited (provided that the considered equivalence relations fulfill stronger demands). In the latter paper Shelah continues the present work.

For any nonzero cardinals $\mu \leq \kappa$ or $\mu=2^{\kappa}$, there is an equivalence relation $\Sigma_{1}^{1}$-definable over $H(\kappa)$ with $\mu$ equivalence classes. There is also a $\Sigma_{1}^{1}$-equivalence relation having $\kappa^{+}$classes (Lemma 3.2). Furthermore, by a simple coding, the possible numbers of equivalence classes of $\Sigma_{1}^{1}$-equivalence relations are closed under unions of length $\leq \kappa$ and products of length $<\kappa$. In other words, assuming that $\gamma \leq \kappa$ and $\chi_{i}, i<\gamma$, are cardinals such that for each $i<\gamma$, there is a $\Sigma_{1}^{1}$-equivalence relation having $\chi_{i}$ equivalence classes, there exists a $\Sigma_{1}^{1}$-equivalence relation having $\bigcup_{i<\gamma} \chi_{i}$ equivalence classes. Similarly, if $\gamma<\kappa$, there also exists a $\Sigma_{1}^{1}$-equivalence relation with $\operatorname{card}\left(\prod_{i<\gamma} \chi_{i}\right)$ equivalence classes (Lemma 3.4).

What are the possible numbers of equivalence classes between $\kappa^{+}$and $2^{\kappa}$ ? The existence of a tree $T \subseteq H(\kappa)$ with $\mu$ branches of length $\kappa$ through it implies that there is a $\Sigma_{1}^{1}$-equivalence relation having $\mu$ equivalence classes (Lemma 3.2). Therefore, the existence of a Kurepa tree of height $\kappa$ with more than $\kappa^{+}$and less than $2^{\kappa}$ branches of length $\kappa$ through it presents an example of a $\Sigma_{1}^{1}$-equivalence relation with many equivalence classes, but not the maximal number. On the other hand, in an ordinary Cohen extension of $\mathrm{L}$, in which $2^{\kappa}>\kappa^{+}$, there is no definable equivalence relation having $\mu$ different equivalence classes when $\kappa^{+}<\mu<2^{\kappa}$ (a proof of this fact is included in the proof of the main theorem, see the comment at the beginning of Subsection 4.4).

We show that, consistency-wise, the closure properties mentioned are the only restrictions concerning the possible numbers of equivalence classes of second order definable equivalence relations (Theorem 1). Namely, the conclusion will be the following: Suppose $\kappa$ satisfies $\kappa^{<\kappa}=\kappa$ and $2^{\kappa}=\kappa^{+}$. Let $\lambda>\kappa^{+}$be a cardinal with $\lambda^{\kappa}=\lambda$ and $\bar{\mu}$ be a fixed sequence of cardinals between $\kappa^{+}$and $\lambda$. Let $P$ denote the forcing adding, for every $\mu \in \bar{\mu}$, a Kurepa tree of height $\kappa$ with $\mu$ branches of length $\kappa$ through it. Then in the generic extension by $P$, there is an equivalence relation which is second order definable over $H(\kappa)$ with $\mu$ equivalence classes if, and only if, $\mu$ is a nonzero cardinal in $\Omega_{\bar{\mu}}$, where $\Omega_{\bar{\mu}}$ is the smallest set containing all the nonzero cardinals $\leq \kappa$ and which is closed under union and product of $\leq \kappa$ cardinals. Note that in this generic extension the possible numbers of equivalence classes of second order definable equivalence relations are determined by the $\Sigma_{1}^{1}$-definable equivalence relations. 
In order to make this paper self-contained, we introduce the standard way to add a Kurepa tree and give some basic facts concerning that forcing (Section 2). The essential points are the following. Firstly, if one adds several new Kurepa trees, the addition of new trees does not produce new $\kappa$-branches of the old trees. Secondly, permutations of the "labels" of the $\kappa$-branches of the generic Kurepa trees determine many different automorphisms of the forcing itself. This kind of automorphism can be used to "copy" two different equivalence classes of a definable equivalence relation to several different equivalence classes. In fact, this way it is possible to show that in a Cohen extension of L, a definable equivalence relation has either at most $\kappa^{+}$ equivalence classes, or the maximal number of equivalence classes, namely $2^{\kappa}$. The main difference to the proof presented in Section 4 is that $\Delta$-lemma cannot be applied in the same straightforward manner as in the standard Cohen case.

In Section 3 we briefly sketch proofs for the basic facts that the possible numbers of equivalence classes of $\Sigma_{1}^{1}$-equivalence relations include all small cardinals and the possible numbers are closed under small unions and products.

The main theorem is stated and proved in Section 4. The proof is divided into several subsections. First in Subsection 4.1 we present a proof for the crucial fact that a second order definable equivalence relation is absolute for generic extensions by the introduced Kurepa tree forcing (Lemma 4.2).

The main ideas in the remaining three subsections are the following. We fix a second order definable equivalence relation $\sim_{\phi, R}$ and consider forcing extensions by the partial order defined in Section 2. The forcing adds $\lambda$ different Kurepa trees. However, we may assume that the forcing name of the parameter has cardinality $\kappa$, and thus, there are only $\kappa$ trees which really have "effect" on the number of classes of the fixed equivalence relation. So we restrict ourselves to the subforcing consisting of the addition of these $\kappa$ "critical" trees. (Note that in Lemma 4.3 we introduce a subforcing consisting of addition of $\kappa^{+}$trees, but right after that in Subsection 4.3, we define "isomorphism classes" of names in order to concentrate only on $\kappa$ generic trees.) Then, as explained in Subsection 4.3, it follows from the assumptions on the cardinal arithmetic that either 1) the fixed equivalence relation has $\chi$ classes, where $\chi$ is a union or product of $\kappa$ cardinals in the prearranged set $\Omega_{\bar{\mu}}$, or otherwise, 2) the number of equivalence classes really depends on $\kappa$ trees, not less than $\kappa$. The latter case is the most difficult and it is presented in Subsection 4.4. There we notice that the fixed equivalence relation must have $\chi$ classes, where $\chi$ is a union of products of cardinals in $\Omega_{\bar{\mu}}$.

In Section 5 we present some remarks. 
2. Adding Kurepa trees. Throughout the paper we assume that $\kappa$ is an uncountable regular cardinal and $\kappa^{<\kappa}=\kappa$. For sets $X$ and $Y$ we denote the set of all functions from $X$ into $Y$ by ${ }^{X} Y$. For a cardinal $\mu$, we let $[X]^{\mu}$ be the set of all subsets of $X$ having cardinality $\mu$.

The following forcing is the "standard" way to add a Kurepa tree [Jec71, Jec97].

Definition 2.1. Let $\mu$ be a cardinal $\geq \kappa$. Define a forcing $P_{\mu}$ as follows. It consists of all pairs $p=\left\langle T^{p},\left\langle b_{\delta}^{p} \mid \delta \in \Delta^{p}\right\rangle\right\rangle$, where

- for some $\alpha<\kappa, T^{p}$ is a subset of $\left\{\eta \mid \eta \in{ }^{\beta} 2\right.$ and $\left.\beta<\alpha\right\}$ of cardinality $<\kappa$ and is closed under restriction;

- $\Delta^{p}$ is a subset of $\mu$ having cardinality $<\kappa$ and each $b_{\delta}^{p}$ is an $\alpha$-branch through $T^{p}$ when $T^{p}$ is ordered by inclusion.

For all $p, q \in P_{\mu}$, we define that $q \leq p$ if

- $T^{q}$ is an end-extension of $T^{p}$;

- $\Delta^{p} \subseteq \Delta^{q}$;

- for every $\delta \in \Delta^{p}, b_{\delta}^{q}$ is an extension of $b_{\delta}^{p}$.

FACT 2.2. (a) $P_{\mu}$ is $\kappa$-closed and (assuming $\kappa^{<\kappa}=\kappa$ ) it satisfies the $\kappa^{+}$-chain condition.

(b) Suppose $G$ is a $P_{\mu}$-generic set over V. In $\mathrm{V}[G], T^{G}=\bigcup_{p \in G} T^{p}$ is a tree of height $\kappa$ and each of its levels has cardinality $<\kappa$.

LeMma 2.3. Let $\widetilde{Q}$ be such that $\mathbf{1} \Vdash_{P_{\mu}}$ " $\widetilde{Q}$ is a $\kappa$-closed forcing notion". Suppose $G$ is a $P_{\mu}$-generic set over $\mathrm{V}$ and $H$ is a $Q$-generic set over $\mathrm{V}[G]$. Then, in $\mathrm{V}[G][H]$, the $\kappa$-branches through the tree $T^{G}=\bigcup_{p \in G} T^{p}$ are the functions $b_{\delta}^{G}, \delta<\mu$, having domain $\kappa$ and such that for every $\alpha<\kappa$, $b_{\delta}^{G}(\alpha)=b_{\delta}^{p}(\alpha)$ for some $p \in G$ with $\delta \in \Delta^{p}$ and $\alpha \in \operatorname{dom}\left(b_{\delta}^{p}\right)$.

Proof. The idea of the proof is the same as in [Jec71]. Suppose $\left\langle p_{0}, \widetilde{q}_{0}\right\rangle$ is a condition in $P_{\mu} * \widetilde{Q}$ and $\widetilde{t}$ is a name such that

$$
\left\langle p_{0}, \widetilde{q}_{0}\right\rangle \Vdash_{P_{\mu} * \widetilde{Q}} \text { " } \widetilde{t} \text { is a } \kappa \text {-branch through } \widetilde{T^{G}} \text { and } \widetilde{t} \notin\left\{\widetilde{b}_{\delta}^{G} \mid \delta<\mu\right\} " .
$$

Since $\mathbf{1} \Vdash_{P_{\mu} * \widetilde{Q}}$ " $\kappa$ is a regular cardinal", it follows that every condition below $\left\langle p_{0}, \widetilde{q}_{0}\right\rangle$ forces that for all $X \in[\mu]^{<\kappa}$ and $\beta<\kappa$, there is $\alpha>\beta$ with

$$
\widetilde{t}(\alpha) \notin\left\{\widetilde{b}_{\delta}^{G}(\alpha) \mid \delta \in X\right\} .
$$

Let $\alpha_{0}$ be the height of $T^{p_{0}}$. Choose conditions $\left\langle p_{n}, \widetilde{q}_{n}\right\rangle$ from $P_{\mu} * \widetilde{Q}$ and ordinals $\alpha_{n}, 1<n<\omega$, so that for every $n<\omega$, the height of the tree $T^{p_{n+1}}$ is greater than $\alpha_{n},\left\langle p_{n+1}, \widetilde{q}_{n+1}\right\rangle \leq\left\langle p_{n}, \widetilde{q}_{n}\right\rangle$, and

$$
\left\langle p_{n+1}, \widetilde{q}_{n+1}\right\rangle \Vdash_{\left(P_{\mu} * \widetilde{Q}\right)} \widetilde{t}\left(\alpha_{n+1}\right) \notin\left\{\widetilde{b}_{\delta}^{G}\left(\alpha_{n+1}\right) \mid \delta \in \Delta^{p_{n}}\right\} .
$$


Define $r$ to be the condition in $P_{\mu}$ satisfying $T^{r}=\bigcup_{n<\omega} T^{p_{n}}, \Delta^{r}=\bigcup_{n<\omega} \Delta^{p_{n}}$, and for every $\delta \in \Delta^{r}, b_{\delta}^{r}=\bigcup_{n \in(\omega \backslash m)} b_{\delta}^{p_{n}}$, where $m$ is the smallest index with $\delta \in \Delta^{p_{m}}$. Then $T^{r}$ is of height $\alpha=\bigcup_{n<\omega} \alpha_{n}$. In order to restrict the $\alpha$ th level of the generic tree, abbreviate the function $\bigcup_{\gamma<\alpha} b_{\delta}^{r}(\gamma), \delta \in \Delta^{r}$, by $f_{\delta}$, and define $r^{\prime}$ to be the condition in $P_{\mu}$ with $T^{r^{\prime}}=T^{r} \cup\left\{f_{\delta} \mid \delta \in \Delta^{r}\right\}$, $\Delta^{r^{\prime}}=\Delta^{r}$, and for every $\delta \in \Delta^{r^{\prime}}$ and $\beta \leq \alpha$,

$$
b_{\delta}^{r^{\prime}}(\beta)= \begin{cases}b_{\delta}^{r}(\beta) & \text { if } \beta<\alpha, \\ f_{\delta} & \text { if } \beta=\alpha .\end{cases}
$$

Now $r^{\prime}$ forces that the $\alpha$ th level of the generic tree $\widetilde{T^{G}}$ consists of the elements $f_{\delta}, \delta \in \Delta^{r^{\prime}}$.

Since $r^{\prime}$ forces $\widetilde{Q}$ to be $\kappa$-closed and $\left\langle\widetilde{q}_{n} \mid n<\omega\right\rangle$ to be a decreasing sequence of conditions, there is $\widetilde{q}^{\prime}$ so that $\left\langle r^{\prime}, q^{\prime}\right\rangle \leq\left\langle p_{n}, \widetilde{q}_{n}\right\rangle$ for every $n<\omega$. Since $\left\langle r^{\prime}, q^{\prime}\right\rangle$ forces that $\widetilde{t}(\alpha) \in\left\{f_{\delta} \mid \delta \in \Delta^{r^{\prime}}\right\}$, there are $\delta \in \Delta^{r^{\prime}}$ and a condition $\left\langle r^{\prime \prime}, \widetilde{q}^{\prime \prime}\right\rangle \leq\left\langle r^{\prime}, \widetilde{q}^{\prime}\right\rangle$ in $P_{\mu} * \widetilde{Q}$ forcing that $\widetilde{t}(\alpha)=f_{\delta}$. However, if $n$ is the smallest index with $\delta \in \Delta^{p_{n}}$, then $\left\langle r^{\prime \prime}, \widetilde{q}^{\prime \prime}\right\rangle$ forces that

$$
\widetilde{t}\left(\alpha_{n+1}\right)=f_{\delta}\left\lceil\alpha_{n+1}=b_{\delta}^{r^{\prime}}\left(\alpha_{n+1}\right)=b_{\delta}^{r^{\prime \prime}}\left(\alpha_{n+1}\right)=\widetilde{b}_{\delta}^{G}\left(\alpha_{n+1}\right),\right.
$$

contrary to $(\mathrm{A})$.

Definition 2.4. Suppose $\lambda>\kappa^{+}$is a cardinal with $\lambda^{\kappa}=\lambda$. Let $\bar{\mu}=$ $\left\langle\mu_{\xi} \mid \xi<\lambda\right\rangle$ be a fixed sequence of cardinals such that $\kappa<\mu_{\xi} \leq \lambda$ and for every $\chi \in\left\{\mu_{\xi} \mid \xi<\lambda\right\} \cup\{\lambda\}$, the set $\left\{\zeta<\lambda \mid \mu_{\zeta}=\chi\right\}$ has cardinality $\lambda$. We define $\mathbb{P}(\bar{\mu})$ to be the product of $P_{\mu_{\xi}}$ forcings:

- $\mathbb{P}(\bar{\mu})$ is the set of all functions $p$ such that $\operatorname{dom}(p)$ is a subset of $\lambda$ with cardinality $<\kappa$, and for every $\xi \in \operatorname{dom}(p), p(\xi)$ is a condition in $P_{\mu_{\xi}}$;

- the order of $\mathbb{P}(\bar{\mu})$ is defined coordinatewise, i.e., for $p, q \in \mathbb{P}(\bar{\mu}), q \leq p$ if $\operatorname{dom}(p) \subseteq \operatorname{dom}(q)$ and for every $\xi \in \operatorname{dom}(p), q(\xi) \leq p(\xi)$.

The weakest condition in $\mathbb{P}(\bar{\mu})$ is the empty function, denoted by $\mathbf{1}$. For each $p \in \mathbb{P}(\bar{\mu})$ and $\xi \in \operatorname{dom}(p)$, we let the condition $p(\xi)$ be the pair $\left\langle T_{\xi}^{p},\left\langle b_{\xi, \delta}^{p} \mid \delta \in \Delta_{\xi}^{p}\right\rangle\right\rangle$. From now on, $\Delta^{p}$ denotes the set $\{\langle\xi, \delta\rangle \mid \xi \in$ $\operatorname{dom}(p)$ and $\left.\delta \in \Delta_{\xi}^{p}\right\}$.

FACT 2.5. (a) The forcing $\mathbb{P}(\bar{\mu})$ is $\kappa$-closed and it has $\kappa^{+}$-c.c.

(b) Suppose $G$ is a $\mathbb{P}(\bar{\mu})$-generic set over $\mathrm{V}$. In $\mathrm{V}[G]$, for every $\xi<\lambda$, the $\kappa$-branches through the tree $T_{\xi}^{G}=\bigcup_{p \in G} T_{\xi}^{p}$ are $\left\{b_{\xi, \delta}^{G} \mid \delta<\mu_{\xi}\right\}$, where each $b_{\xi, \delta}^{G}$ is the function

$$
\bigcup\left\{b_{\xi, \delta}^{p} \mid p \in G, \xi \in \operatorname{dom}(p) \text { and } \delta \in \Delta_{\xi}^{p}\right\} .
$$

Proof. (b) Since $\mathbf{1} \Vdash_{\mathbb{P}(\bar{\mu} \uparrow(\xi+1))}$ " $\mathbb{P}(\bar{\mu} \uparrow(\kappa \backslash(\xi+1)))$ is $\kappa$-closed", the claim follows from Lemma 2.3 . 
Definition 2.6. For all $\mathbb{P}(\bar{\mu})$-names $\tau$, define

$$
\Delta^{\tau}=\bigcup\left\{\Delta^{p} \mid \text { condition } p \text { appears in } \tau\right\} .
$$

Let $\Delta_{1 s t}^{\tau}$ denote the set $\left\{\xi \mid\langle\xi, \delta\rangle \in \Delta^{\tau}\right\}$, and $\Delta_{\xi}^{\tau}$ the set $\left\{\delta \mid\langle\xi, \delta\rangle \in \Delta^{\tau}\right\}$.

Definition 2.7. We denote by $\operatorname{Sqs}(\bar{\mu})$ the set of all sequences $\bar{z}=\left\langle z_{\xi}\right|$ $\xi \in Z\rangle$ such that $Z \subseteq \lambda$ and for each $\xi \in Z, z_{\xi}$ is a subset of $\mu_{\xi}$ of cardinality at least $\kappa$. In order to keep our notation coherent, let $\Delta^{\bar{z}}$ be a shorthand for the set $\bigcup_{\xi \in Z}\{\xi\} \times z_{\xi}$. For every $\bar{z} \in \operatorname{Sqs}(\bar{\mu})$ define

$$
\mathbb{P}(\bar{z})=\left\{p \in \mathbb{P}(\bar{\mu}) \mid \Delta^{p} \subseteq \Delta^{\bar{z}}\right\}
$$

A forcing $Q$ is a complete subforcing of $P$ if every maximal antichain in $Q$ is also a maximal antichain in $P$ (a set $X$ of conditions is an antichain in $Y$ if all $p \neq q$ in $X$ are incompatible, i.e., there is no $r \in Y$ with $r \leq p, q)$. The following basic facts are needed later on.

FACT 2.8. (a) Every subforcing $\mathbb{P}(\bar{z})$ with $\bar{z} \in \operatorname{Sqs}(\bar{\mu})$ is a complete subforcing of $\mathbb{P}(\bar{\mu})$.

(b) For every $p \in \mathbb{P}(\bar{\mu})$, the restriction $\{q \in \mathbb{P}(\bar{\mu}) \mid q \leq p\}$ is a forcing notion which is equivalent to $\mathbb{P}(\bar{\mu})$.

The following two definitions will be our main tools. Namely, every permutation $\pi$ of the indices of the labels of the branches in the generic trees added by $\mathbb{P}(\bar{\mu})$ determines an automorphism $\widehat{\pi}$ of $\mathbb{P}(\bar{\mu})$. This means that for every condition $p$ in $\mathbb{P}(\bar{\mu})$ and $\mathbb{P}(\bar{\mu})$-name $\tau$ there are many "isomorphic" copies of $p$ and $\tau$ inside $\mathbb{P}(\bar{\mu})$. Naturally, the copies $\widehat{\pi}(p)$ and $\widehat{\pi}(\tau)$ of $p$ and $\tau$, respectively, satisfy all the same formulas (see (2.1) below).

Definition 2.9. We define $\operatorname{Mps}(\bar{\mu})$ to be the set of all mappings $\pi$ which can be defined as follows. The domain of $\pi$ is $\Delta^{\bar{y}}$ for some $\bar{y}=\left\langle y_{\xi} \mid \xi \in Y\right\rangle$ in $\operatorname{Sqs}(\bar{\mu})$. In addition, there exists an injective function $\pi_{1 s t}$ from $Y$ into $\lambda$ and injective functions $\pi_{\xi}$ from $y_{\xi}$ into $\mu_{\xi}$, for all $\xi \in Y$, such that for all $\langle\xi, \delta\rangle \in \operatorname{dom}(\pi)$

$$
\pi\langle\xi, \delta\rangle=\left\langle\pi_{1 s t}(\xi), \pi_{\xi}(\delta)\right\rangle
$$

For every $\bar{z} \in \operatorname{Sqs}(\bar{\mu}), \operatorname{Mps}(\bar{z})$ is the collection $\left\{\pi \in \operatorname{Mps}(\bar{\mu}) \mid \operatorname{dom}(\pi) \subseteq \Delta^{\bar{z}}\right\}$.

Definition 2.10. For every $p \in \mathbb{P}(\bar{\mu})$ and $\pi \in \operatorname{Mps}(\bar{\mu})$ with $\Delta^{p} \subseteq$ $\operatorname{dom}(\pi)$, we let $\pi(p)$ denote the condition $q$ in $\mathbb{P}(\bar{\mu})$ for which

- $\operatorname{dom}(q)=\pi_{1 s t}[\operatorname{dom}(p)]$;

- for every $\zeta \in \operatorname{dom}(q), T_{\zeta}^{q}=T_{\xi}^{p}$ and $\Delta_{\zeta}^{q}=\pi_{\xi}\left[\Delta_{\xi}^{p}\right]$, where $\xi=\left(\pi_{1 s t}\right)^{-1}(\zeta)$;

- for every $\langle\zeta, \varepsilon\rangle \in \Delta^{q}, b_{\zeta, \varepsilon}^{q}=b_{\xi, \delta}^{p}$, where $\langle\xi, \delta\rangle=\pi^{-1}\langle\zeta, \varepsilon\rangle$.

When $\tau$ is a $\mathbb{P}(\bar{z})$-name and $\pi$ a mapping in $\operatorname{Mps}(\bar{\mu})$ with $\Delta^{\tau} \subseteq \operatorname{dom}(\pi)$, $\pi(\tau)$ denotes the $\mathbb{P}(\bar{z})$-name which is the result of recursively replacing every 
condition $p$ in $\tau$ with $\pi(p)$, i.e.,

$$
\pi(\tau)=\{\langle\pi(\sigma), \pi(p)\rangle \mid\langle\sigma, p\rangle \in \tau\} .
$$

Analogously, for sequences $\bar{z}=\left\langle z_{\xi} \mid \xi \in Z\right\rangle$ with $\Delta^{\bar{z}} \subseteq \operatorname{dom}(\pi)$, we let $\pi(\bar{z})$ denote the sequence $\left\langle z_{\zeta}^{\prime} \mid \zeta \in Z^{\prime}\right\rangle$, where $Z^{\prime}=\pi_{1 s t}[Z]$ and for each $\zeta \in Z^{\prime}$, $z_{\zeta}^{\prime}=\pi_{\xi}\left[z_{\xi}\right]$ with $\xi=\left(\pi_{1 s t}\right)^{-1}(\zeta)$.

FACT 2.11. For every subforcing $\mathbb{P}(\bar{z})$ and $\pi \in \operatorname{Mps}(\bar{z})$ with $\operatorname{dom}(\pi)=$ $\Delta^{\bar{z}}$, the mapping $p \mapsto \pi(p)$ is an isomorphism between $\mathbb{P}(\bar{z})$ and $\mathbb{P}(\pi(\bar{z}))$.

Suppose $\mathbb{P}(\bar{z})$ is a subforcing of $\mathbb{P}(\bar{\mu})$. The isomorphism determined by some $\pi \in \operatorname{Mps}(\bar{z})$ is denoted by $\widehat{\pi}$. It follows that if $\operatorname{dom}(\pi)=\Delta^{\bar{z}}, p \in \mathbb{P}(\bar{z})$, $\psi\left(x_{1}, \ldots, x_{n}\right)$ with $n<\omega$ is a formula, and $\tau_{1}, \ldots, \tau_{n}$ are $\mathbb{P}(\bar{z})$-names then

$$
p \Vdash_{\mathbb{P}(\bar{z})} \psi\left(\tau_{1}, \ldots, \tau_{n}\right) \quad \text { iff } \quad \widehat{\pi}(p) \Vdash_{\mathbb{P}(\pi(\bar{z}))} \psi\left(\widehat{\pi}\left(\tau_{1}\right), \ldots, \widehat{\pi}\left(\tau_{n}\right)\right) .
$$

In particular, a mapping $\pi$ in $\operatorname{Mps}(\bar{z})$ determines an automorphism of $\mathbb{P}(\bar{z})$ when $\pi_{1 s t}$ is a permutation of $Z$ and each $\pi_{\xi}$ is a bijection from $z_{\xi}$ onto $z_{\pi_{1 s t}(\xi)}$

3. Basic facts on $\Sigma_{1}^{1}$-equivalence relations. Recall that we assume $\kappa$ to be an uncountable regular cardinal. We let $H(\kappa)$ denote the set of all sets having transitive closure of cardinality $<\kappa$.

Definition 3.1. We say that $\phi$ defines an equivalence relation $\sim_{\phi, R}$ on ${ }^{\kappa} 2$ with a parameter $R \subseteq H(\kappa)$ when

- $\phi$ is a second order sentence in the vocabulary consisting of $\epsilon$, one unary relation symbol $S_{0}$, and binary relation symbols $S_{1}$ and $S_{2}$;

- the following definition gives an equivalence relation on ${ }^{\kappa} 2$ : for all $f, g \in{ }^{\kappa} 2$,

$$
f \sim_{\phi, R} g \quad \text { iff } \quad\langle H(\kappa), \in, R, f, g\rangle \models \phi,
$$

where $R, f$, and $g$ are the interpretations of the symbols $S_{0}, S_{1}$, and $S_{2}$ respectively.

An equivalence relation is $\Sigma_{1}^{1}$-definable if $\phi$ defines it and $\phi$ is of the form $(\exists X)\left(\psi\left(S_{0}, S_{1}, S_{2}, X\right)\right)$, where $X$ is the only second order variable appearing in $\psi$.

We abbreviate $\operatorname{card}\left(\left\{f / \sim_{\phi, R} \mid f \in{ }^{\kappa} 2\right\}\right)$ by $\operatorname{No}\left(\sim_{\phi, R}\right)$.

LEMMA 3.2. (a) For every nonzero cardinal $\mu \in \kappa \cup\left\{\kappa, 2^{\kappa}\right\}$, there exists a $\Sigma_{1}^{1}$-equivalence relation $\sim_{\phi, R}$ on ${ }^{\kappa} 2$ with $\operatorname{No}\left(\sim_{\phi, R}\right)=\mu$.

(b) There is a $\Sigma_{1}^{1}$-equivalence relation $\sim_{\phi, R}$ on ${ }^{\kappa} 2$ with $\operatorname{No}\left(\sim_{\phi, R}\right)=\kappa^{+}$.

(c) If $T$ is a tree with $\operatorname{card}(T)=\kappa$, then there exists a $\Sigma_{1}^{1}$-equivalence relation $\sim_{\phi, R}$ on ${ }^{\kappa} 2$ with $\operatorname{No}\left(\sim_{\phi, R}\right)=\operatorname{card}\left(\operatorname{Br}_{\kappa}(T)\right)+1$.

Proof. Let $\varrho$ be a fixed definable bijection from $\kappa$ onto $\kappa \times \kappa$. For a binary relation $R$, we denote the set $\{\varrho(\xi) \mid$ for some $\xi<\kappa,\langle\xi, 1\rangle \in R\}$ by $\varrho(R)$. 
(a) In the cases $\mu \in \kappa \cup\{\kappa\}$, the parameter can code a list of $\mu$ nonequivalent functions. In the case $\operatorname{No}\left(\sim_{\phi, R}\right)=2^{\kappa}$ all the functions in ${ }^{\kappa} 2$ can be nonequivalent.

(b) A sentence $\phi\left(R_{1}, R_{2}, R_{3}\right)$ saying

"(both $\varrho\left(R_{1}\right)$ and $\varrho\left(R_{2}\right)$ are well-orderings of $\kappa$, and $\varrho\left(R_{3}\right)$ is an isomorphism between them) or (neither $\varrho\left(R_{1}\right)$ nor $\varrho\left(R_{2}\right)$ is a wellordering of $\kappa$ )"

defines a $\Sigma_{1}^{1}$-equivalence relation as required.

(c) We may assume, without loss of generality, that the elements of $T$ are ordinals below $\kappa$. Using $\langle T,<\rangle$ as a parameter, let a sentence $\phi\left(R_{0}, R_{1}, R_{2}\right)$ say that

" $\left(\varrho\left(R_{1}\right)=\varrho\left(R_{2}\right)\right.$ is a $\kappa$-branch in $\left.R_{0}\right)$ or (neither $\varrho\left(R_{1}\right)$ nor $\varrho\left(R_{2}\right)$ is a $\kappa$-branch in $R_{0}$ )".

Then $\left(\exists R_{2}\right)\left(\phi\left(R_{0}, R_{1}, R_{2}\right)\right)$ defines a $\Sigma_{1}^{1}$-equivalence relation as desired.

Conclusion 3.3. Let $G$ be a $\mathbb{P}(\bar{\mu})$-generic set over $\mathrm{V}$. Then in $\mathrm{V}[G]$, for every nonzero cardinal $\chi$ in $\kappa \cup\left\{\kappa, \kappa^{+}, 2^{\kappa}\right\} \cup\left\{\mu_{\xi} \mid \xi<\lambda\right\}$, there exists a $\Sigma_{1}^{1}$-equivalence relation $\sim_{\phi, R}$ with $\operatorname{No}\left(\sim_{\phi, R}\right)=\chi$.

Proof. The claim follows from Fact 2.5 together with Lemma 3.2.

In the next section we shall need the following properties of $\Sigma_{1}^{1}$-equivalence relations.

Lemma 3.4. Suppose $\gamma \leq \kappa$ and $\chi_{i}, i<\gamma$, are nonzero cardinals such that $\phi_{i}$ defines a $\Sigma_{1}^{1}$-equivalence relation on ${ }^{\kappa} 2$ with the parameter $R_{i}$ and it has $\chi_{i}$ equivalence classes.

(a) There exists a $\Sigma_{1}^{1}$-equivalence relation $\sim_{\phi, R}$ on ${ }^{\kappa} 2$ with $\operatorname{No}\left(\sim_{\phi, R}\right)=$ $\bigcup_{i<\gamma} \chi_{i}$.

(b) There exists a $\Sigma_{1}^{1}$-equivalence relation $\sim_{\phi, R}$ on ${ }^{\kappa} 2$ with $\operatorname{No}\left(\sim_{\phi, R}\right)=$ $\operatorname{card}\left(\prod_{i<\gamma} \chi_{i}\right)$.

Proof. Both claims are simple corollaries of the fact that there are a parameter $R \subseteq H(\kappa)$ and a formula $\psi(x)$ such that for all $f, g, h \in{ }^{\kappa} 2$,

$$
\langle H(\kappa), \in, R, f, g, h\rangle \models \psi(i)
$$

if, and only if,

$$
\langle H(\kappa), \in, R[i], f[i], g[i], h[i]\rangle=\phi_{i},
$$

where $R[i], f[i], g[i]$, and $h[i]$ are the $i$ th parts of $R, f, g$, and $h$ respectively, in some definable coding. Furthermore $R[i]=R_{i}$ for every $i<\gamma$. 
4. Possible numbers of equivalence classes. Our goal is to show the consistency of the claim: the closure under unions and products in Lemma 3.4 are the only restrictions on the possible numbers of equivalence classes of equivalence relations on $2^{\kappa}$ which are second order definable over $H(\kappa)$.

The following notation is used in the theorem.

Definition 4.1. Suppose $\bar{\mu}=\left\langle\mu_{\xi} \mid \xi<\lambda\right\rangle$ is a sequence of cardinals. Define $\Omega_{\bar{\mu}}$ to be the smallest set of cardinals such that

- every nonzero cardinal $\leq \kappa^{+}$is in $\Omega_{\bar{\mu}}$;

- $\left\{\mu_{\xi} \mid \xi<\lambda\right\} \subseteq \Omega_{\bar{\mu}}$

- if $\gamma \leq \kappa$ and $\chi_{i}, i<\gamma$, are cardinals in $\Omega_{\bar{\mu}}$, then both $\bigcup_{i<\gamma} \chi_{i}$ and $\operatorname{card}\left(\prod_{i<\gamma} \chi_{i}\right)$ are in $\Omega_{\bar{\mu}}$.

TheOrem 1. Suppose that

- $\kappa$ is an uncountable cardinal with $\kappa^{<\kappa}=\kappa$ and $2^{\kappa}=\kappa^{+}$;

- $\lambda>\kappa^{+}$is a cardinal with $\lambda^{\kappa}=\lambda$;

- $\bar{\mu}=\left\langle\mu_{\xi} \mid \xi<\lambda\right\rangle$ and $\mathbb{P}(\bar{\mu})$ are as in Definition 2.4;

- $\Omega_{\bar{\mu}}$ is as in Definition 4.1;

- $\chi^{\gamma} \leq \chi^{+}$for every $\chi \in \Omega_{\bar{\mu}}$ with $\chi>\kappa^{+}$and $\gamma<\kappa$.

Then for every $\mathbb{P}(\bar{\mu})$-generic set $G$, the extension $\mathrm{V}[G]$ has the property that all cardinals and cofinalities are preserved, there are no new sets of cardinality $<\kappa, 2^{\kappa}=\lambda$ and for all cardinals $\chi$, the following conditions are equivalent:

(A) $\quad \chi \in \Omega_{\bar{\mu}}$;

(B) there is a sentence $\psi$ defining a $\Sigma_{1}^{1}$-equivalence relation $\sim_{R, \psi}$ on ${ }^{\kappa} 2$ with a parameter $R \subseteq H(\kappa)$ such that the number of equivalence classes of $\sim_{R, \psi}$ is $\chi$;

(C) there is a second order sentence $\phi$ defining over $H(\kappa)$ an equivalence relation $\sim_{\phi, R}$ on ${ }^{\kappa} 2$ with a parameter $R$ such that the number of equivalence classes of $\sim_{\phi, R}$ is $\chi$.

REMARK. Because $\mathbb{P}(\bar{\mu})$ does not add new subsets of cardinality $<\kappa$, the definition of $\Omega_{\bar{\mu}}$ yields the same sets in the ground model and in the generic extension.

The rest of this section is devoted to the proof of this theorem. Because of Conclusion 3.3 and Lemma 3.4 it remains to show that if $\widetilde{R}$ is a $\mathbb{P}(\bar{\mu})$-name for a subset of $H(\kappa)$ and $\phi$ is a second order sentence such that in every generic extension, $\phi$ defines over $H(\kappa)$ an equivalence relation $\sim_{\phi, R}$ on $2^{\kappa}$, then

$$
\mathbf{1} \Vdash_{\mathbb{P}(\bar{\mu})} \operatorname{No}\left(\sim_{\phi, \tilde{R}}\right) \in \Omega_{\bar{\mu}}
$$


By Fact $2.8($ b), we may assume that $\widetilde{R}$ is chosen so that for a fixed cardinal $\theta$ the following holds:

$$
\mathbf{1} \Vdash_{\mathbb{P}(\bar{\mu})} \operatorname{No}\left(\sim_{\phi, \tilde{R}}\right)=\theta .
$$

Without loss of generality the name $\widetilde{R}$ has cardinality $\kappa$. Of course we may assume that $\theta>\kappa^{+}$, otherwise the claim follows from Lemma 3.2.

We prove that $\theta$ has the desired form, i.e., it is in $\Omega_{\bar{\mu}}$. The first thing to show is that $\operatorname{No}\left(\sim_{\phi, \tilde{R}}\right)$ depends only on the coordinates $\xi$ appearing in $\widetilde{R}$ with $\mu_{\xi} \leq \theta$ in the following way: there exists a "small" subforcing $\mathbb{P}(\bar{z})$ of $\mathbb{P}(\bar{\mu})\left(\operatorname{dom}(\bar{z})\right.$ has cardinality $\kappa^{+}$and the union of the sets in $\operatorname{ran}(\bar{z})$ has cardinality $\theta$ ) so that $\phi$ and $\widetilde{R}$ yield an equivalence relation with $\theta$ classes already in $\mathrm{V}^{\mathbb{P}(\bar{z})}$. This will be an application of Lemma 4.2, which provides the fact that the truth of a second order sentence in $H(\kappa)$ is absolute between a middle extension $\mathrm{V}^{\mathbb{P}(\bar{y})}$ and the full extension $\mathrm{V}^{\mathbb{P}(\bar{\mu})}$. The formal details are presented in Subsection 4.1.

How the small subforcing is applied? We are going to fix a sequence $\bar{z}$ as in Lemma 4.3 and a list $\left\langle\sigma_{\alpha} \mid \alpha<\theta\right\rangle$ of $\mathbb{P}(\bar{z})$-names for representatives of different $\sim_{\phi, \tilde{R}}$ classes. From the choice of the small $\bar{z}$ it follows that the number of nonisomorphic names in the fixed list is at most $\kappa^{+}$. Here an isomorphism class of $\sigma_{\alpha}$, roughly speaking, consists of all names $\sigma_{\beta}$ which are images of $\sigma_{\alpha}$ under some $\pi \in \operatorname{Mps}(\bar{z})$ fixing $\widetilde{R}$. Because $\theta>\kappa^{+}, \theta$ must be the supremum of the cardinalities of the isomorphism classes of the names $\sigma_{\alpha}, \alpha<\theta$. Hence to study what form $\theta$ has, it suffices to look at what the cardinalities of the isomorphism classes of $\sigma_{\alpha}$ 's are. At the beginning of Subsection 4.2 this is explained more formally.

So we fix a name $\sigma_{\alpha^{*}}$ from the list of representatives and consider the cardinality of the isomorphism class of this name. The study is divided into two parts presented in Subsections 4.3 and 4.4. Firstly, we assume that the number of so called critical indices is strictly less than $\kappa$. From the assumption that $\chi^{\gamma} \leq \chi^{+}$holds for every $\chi \in \Omega_{\bar{\mu}} \backslash \kappa^{+}$and $\gamma<\kappa$, it follows that the cardinality of the isomorphism class of $\sigma_{\alpha^{*}}$ is a small product or a small union of cardinals in $\Omega_{\bar{\mu}}$.

Secondly, we assume that the number of critical indices is $\kappa$. Our assumption on cardinal arithmetic in the ground model implies that all the products of less than $\kappa$ "critical cardinals" have smaller cardinality than the cardinality of the isomorphism class under consideration. (Note that by the assumption $\kappa^{<\kappa}=\kappa$, the number of such products is $\kappa$.) So the cardinality of the fixed isomorphism class is at least the supremum of all this type of products. On the other hand, the cardinality of the isomorphism class cannot exceed this union. A reason for this is that if the cardinality of the isomorphism class is even larger than the supremum of all small products, then there are, by the $\Delta$-lemma, "coherent names" for nonequivalent 
functions in the isomorphism class (Lemma 4.9). Roughly speaking, such coherent names can be "copied" by automorphisms of $\mathbb{P}(\bar{z})$ (fixing the name for a parameter). This yields more than $\theta$ names for nonequivalent functions. To prove all the details, we introduce some technical tools at the beginning of Subsection 4.4.

4.1. Choice of a small subforcing. In this subsection we prove that there is a subforcing $\mathbb{P}(\bar{z})$ of $\mathbb{P}(\bar{\mu})$ such that the cardinality of $\mathbb{P}(\bar{z})$ is $\theta$, there are at most $\kappa^{+}$coordinates in $\mathbb{P}(\bar{z})$, and already $\mathbb{P}(\bar{z})$ produces $\theta$ different equivalent classes of $\sim_{\phi, \tilde{R}}$.

As mentioned above, the first lemma will play a central role in the proof of the main lemma of this subsection, Lemma 4.3.

For a regular cardinal $\chi$, let $\operatorname{Col}\left(\kappa^{+}, \lambda\right)$ denote the standard $\kappa^{+}$-closed forcing notion collapsing the cardinality of $\lambda$ to $\kappa^{+}$.

Lemma 4.2. $\left({ }^{1}\right)$ Suppose $\bar{z} \in \operatorname{Sqs}(\bar{\mu})$ is such that $\operatorname{dom}(\bar{z})$ and each element in $\operatorname{ran}(\bar{z})$ have cardinality $\geq \kappa^{+}$.

(a) Assume that $\bar{y} \in\{\bar{z}, \bar{\mu}\}, q \in \mathbb{P}(\bar{y})$, and $\tau_{1}, \ldots, \tau_{n}$ are $\mathbb{P}(\bar{y})$-names with $n<\omega$ and $q \Vdash_{\mathbb{P}(\bar{y})} \tau_{1}, \ldots, \tau_{n} \subseteq H(\kappa)$. Then for all $\operatorname{Col}\left(\kappa^{+}, \lambda\right)$-generic filters $K$ and for all second order sentences $\phi$ in vocabulary $\left\{\in, R_{1}, \ldots, R_{n}\right\}$,

$$
\begin{aligned}
&\left(q \Vdash_{\mathbb{P}(\bar{y})}\left\langle H(\kappa), \in, \tau_{1}, \ldots, \tau_{n}\right\rangle \models \phi\right)^{\mathrm{V}} \\
& \text { iff } \quad\left(q \Vdash_{\mathbb{P}(\bar{y})}\left\langle H(\kappa), \in, \tau_{1}, \ldots, \tau_{n}\right\rangle \models \phi\right)^{\mathrm{V}[K]} .
\end{aligned}
$$

(b) Suppose $\sigma$ is a $\mathbb{P}(\bar{z})$-name, $\mathbf{1} \Vdash_{\mathbb{P}(\bar{z})} \sigma \subseteq H(\kappa), \pi$ is a mapping in $\operatorname{Mps}(\bar{\mu})$ such that $\operatorname{card}(\pi) \leq \kappa, \operatorname{ran}(\pi) \subseteq \Delta^{\bar{z}}$, and $\pi$ is identity on $\operatorname{dom}(\pi) \cap$ $\Delta^{\sigma}$. Let $p$ be a condition in $\mathbb{P}(\bar{\mu})$ and $\tau$ a $\mathbb{P}(\bar{\mu})$-name such that $p \Vdash_{\mathbb{P}(\bar{\mu})}$ $\tau \subseteq H(\kappa)$ (we can assume both $\sigma$ and $\tau$ have cardinality $\leq \kappa)$. Then there exists $\varrho$ in $\operatorname{Mps}(\bar{\mu})$ of cardinality $\leq \kappa$ extending $\pi$ such that the domain of $\varrho$ contains $\Delta^{p} \cup \Delta^{\sigma} \cup \Delta^{\tau}, \operatorname{ran}(\varrho) \subseteq \Delta^{\bar{z}}, \varrho$ is identity on $\Delta^{\sigma}$, and for all second order sentences $\phi$ in vocabulary $\left\{\in, R_{1}, R_{2}\right\}$,

$$
\begin{aligned}
& p \Vdash_{\mathbb{P}(\bar{\mu})}\langle H(\kappa), \in, \sigma, \tau\rangle \models \phi \\
& \text { iff } \quad \varrho(p) \Vdash_{\mathbb{P}(\bar{z})}\langle H(\kappa), \in, \sigma, \varrho(\tau)\rangle \models \phi .
\end{aligned}
$$

(c) For every $\mathbb{P}(\bar{\mu})$-generic set $H$ over $\mathrm{V}, G=H \cap \mathbb{P}(\bar{z})$ is a $\mathbb{P}(\bar{z})$-generic set over $\mathrm{V}, \mathrm{V}[G] \subseteq \mathrm{V}[H]$, and for all $(R \subseteq H(\kappa))^{\mathrm{V}}, f, g \in\left({ }^{\kappa} 2\right)^{\mathrm{V}[G]}$, and second order sentences $\phi$ in vocabulary $\left\{\in, R_{1}, R_{2}, R_{3}\right\}$,

$$
\begin{aligned}
& (\langle H(\kappa), \in, R, f, g\rangle \models \phi)^{\mathrm{V}[G]} \\
\text { iff } \quad(\langle H(\kappa), \in, R, f, g\rangle & =\phi)^{\mathrm{V}[H]} .
\end{aligned}
$$

$\left({ }^{1}\right)$ The authors wish to thank the anonymous referee for pointing out that instead of $\Sigma_{1}^{1}$-definable equivalence relations one can consider arbitrary second order definable equivalence relations. 
Proof. (a) Recall that we assume $\kappa^{<\kappa}=\kappa$ and hence $\mathbb{P}(\bar{y})$ has $\kappa^{+}$-c.c. in V. Since $\operatorname{Col}\left(\kappa^{+}, \lambda\right)$ is $\kappa^{+}$-closed, cardinals $\leq \kappa^{+}$are preserved, $(\mathbb{P}(\bar{y}))^{\mathrm{V}}=$ $(\mathbb{P}(\bar{y}))^{\mathrm{V}[K]}$, and $\left(H\left(\kappa^{+}\right)\right)^{\mathrm{V}[K]}=\left(H\left(\kappa^{+}\right)\right)^{\mathrm{V}}$. Moreover, $\kappa^{<\kappa}=\kappa$ holds in $\mathrm{V}[K]$, and hence $\mathbb{P}(\bar{y})$ has $\kappa^{+}$-c.c. in $\mathrm{V}[K]$ too.

For any $\mathbb{P}(\bar{y})$-name $\sigma$ and $q \in \mathbb{P}(\bar{y})$, if $q \Vdash_{\mathbb{P}(\bar{y})} \sigma \subseteq H(\kappa)$, then there exists a "nice" $\mathbb{P}(\bar{y})$-name $\tau$ such that $\operatorname{card}(\tau) \leq \kappa$ and $q \Vdash_{\mathbb{P}(\bar{y})} \sigma=\tau$. So for any $\mathbb{P}(\bar{y})$-name $\sigma$ in $\mathrm{V}[K]$ and $q \in \mathbb{P}(\bar{y})$ with $q \Vdash_{\mathbb{P}(\bar{y})} \sigma \subseteq H(\kappa)$, there exists a $\mathbb{P}(\bar{y})$-name $\tau$ in $\mathrm{V}$ with $q \Vdash_{\mathbb{P}(\bar{y})} \sigma=\tau$.

By the preservation of $\kappa^{+}$-c.c., for any $A \subseteq \mathbb{P}(\bar{y}), A$ is an antichain in $\mathrm{V}$ iff $A$ is an antichain in $\mathrm{V}[K]$. But the definition of $q \Vdash_{\mathbb{P}(\bar{y})}\left\langle H(\kappa), \in, \sigma_{1}, \ldots, \sigma_{n}\right\rangle$ $\models \phi$ depends only on possible antichains of $\mathbb{P}(\bar{y})$ and possible nice names for subsets of $H(\kappa)$. Hence the claim follows from the fact that $\mathrm{V}$ and $\mathrm{V}[K]$ have the same antichains and the same nice names for subsets of $H(\kappa)$.

(b) Suppose that $\left(p \Vdash_{\mathbb{P}(\bar{\mu})}\langle H(\kappa), \in, \sigma, \tau\rangle \models \phi\right)^{\mathrm{V}}$ holds. By (a), $\left(p \Vdash_{\mathbb{P}(\bar{\mu})}\right.$ $\langle H(\kappa), \in, \sigma, \tau\rangle=\phi)^{\mathrm{V}[K]}$ holds. Inside the generic extension $\mathrm{V}[K]$ there exists a mapping $\iota$ in $\operatorname{Mps}(\bar{\mu})$ extending $\pi$ such that $\iota_{1 s t}$ is a bijection from $\operatorname{dom}(\bar{\mu})=\lambda$ into $\operatorname{dom}(\bar{z})$ which is identity on $\Delta_{1 s t}^{\sigma}$. Moreover, for every $\xi \in$ $\operatorname{dom}(\bar{z}), \iota_{\xi}$ is a bijection from $\mu_{\xi}$ into $z_{\xi}$ which is identity on $\Delta_{\xi}^{\sigma}$ (all the cardinals between $\kappa^{+}$and $\lambda^{+}$are collapsed to $\left.\kappa^{+}\right)$. Then $\iota$ determines an isomorphism between $\mathbb{P}(\bar{\mu})$ and $\mathbb{P}(\bar{z})$. Therefore, $\left(\iota(p) \Vdash_{\mathbb{P}(\bar{z})}\langle H(\kappa), \in, \iota(\sigma), \iota(\tau)\rangle=\right.$ $\phi)^{\mathrm{V}[K]}$ holds. Choose $\varrho \subseteq \iota$ so that $\operatorname{card}(\varrho) \leq \kappa$ and $\Delta^{p} \cup \Delta^{\sigma} \cup \Delta^{\tau} \subseteq \operatorname{dom}(\varrho)$. Note that $\varrho$ is in $\mathrm{V}$. Now $\left(\varrho(p) \Vdash_{\mathbb{P}(\bar{z})}\langle H(\kappa), \in, \sigma, \varrho(\tau)\rangle \models \phi\right)^{\mathrm{V}[K]}$ holds, and by $\left(\right.$ a), $\left(\varrho(p) \Vdash_{\mathbb{P}(\bar{\mu})}\langle H(\kappa), \in, \sigma, \varrho(\tau)\rangle=\phi\right)^{\mathrm{V}}$ holds.

The other direction follows in the same manner from (a).

(c) Let $\widetilde{R}, \widetilde{f}$, and $\widetilde{g}$ be $\mathbb{P}(\bar{z})$-names for $R, f$, and $g$ respectively.

If $p \in G$ is a condition forcing the left hand side to be true, then $p$ is in $H$ and $p$ forces the right hand side to be true, since in (b) one may choose $\pi$ to be identity on $\Delta^{p} \cup \Delta^{\widetilde{R}} \cup \Delta^{\widetilde{f}} \cup \Delta^{\widetilde{g}}$.

Suppose $p \in H$ is a condition forcing the right hand side to be true. By (b) there is a condition $q \in \mathbb{P}(\bar{z})$ forcing the left hand side to be true and $q$ is determined by a map $\varrho$ in $\operatorname{Mps}(\bar{\mu})$ which is identity on $\Delta^{\widetilde{R}} \cup \Delta^{\widetilde{f}} \cup \Delta^{\widetilde{g}}$. The only small problem is that we should have $q \in G$. However, the set of $q$ 's like that is predense below $p$ (i.e. each $r \leq p$ is compatible with some $q$ like that). The reason for this is that for any $r \in \mathbb{P}(\bar{\mu})$ with $r \leq p, r$ and $\varrho(r)$ are compatible, provided that for every $\langle\xi, \delta\rangle \in \Delta^{r}$, either $\varrho(\xi, \delta)=\langle\xi, \delta\rangle$ or $\varrho(\xi, \delta) \notin \Delta^{r}$. So given arbitrary $r \leq p$, both $\pi$ and $\varrho$ in (b) can be chosen so that $r$ and $\varrho(r)$ are compatible. Therefore there must be some $r \leq p$ and $\varrho$ fixing $\widetilde{R}, \widetilde{f}$, and $\widetilde{g}$ such that $\varrho(r) \in H$. Hence $\varrho(r)$ is in $G$.

REMARK. Even though the use of $\operatorname{Col}\left(\kappa^{+}, \lambda\right)$ provided an easy proof of the previous lemma, the same idea cannot be applied in the proof of the next 
lemma: $\theta$ might be a singular cardinal of cofinality $\aleph_{0}$ and hence $\operatorname{Col}(\theta, \lambda)$ is not even $\aleph_{1}$-closed.

Lemma 4.3. Recall that we assume $\theta>\kappa^{+}$and (4.1) holds, i.e.,

$$
\mathbf{1} \Vdash_{\mathbb{P}(\bar{\mu})} \operatorname{No}\left(\sim_{\phi, \tilde{R}}\right)=\theta .
$$

Suppose $\mathbb{P}(\bar{z})$ is a subforcing of $\mathbb{P}(\bar{\mu})$ such that

- $\bar{z}=\left\langle z_{\xi} \mid \xi \in Z\right\rangle$;

- $Z$ is a subset of $\lambda$ satisfying $\operatorname{card}(Z)=\kappa^{+}$and $\Delta_{1 s t}^{\widetilde{R}} \subseteq Z$;

- for each $\xi \in \Delta_{1 s t}^{\widetilde{R}}, z_{\xi}=\mu_{\xi}$ if $\mu_{\xi} \leq \theta$, and $z_{\xi} \in\left\{y \in\left[\mu_{\xi}\right]^{\kappa^{+}} \mid \Delta_{\xi}^{\widetilde{R}} \subseteq y\right\}$ otherwise.

- $Z \backslash \Delta_{1 s t}^{\widetilde{R}}$ is of cardinality $\kappa^{+}$(follows from the choice of $\widetilde{R}$ );

- for every $\xi \in Z \backslash \Delta_{1 s t}^{\widetilde{R}}, \mu_{\xi}>\theta$ and $z_{\xi}$ is some set in $\left[\mu_{\xi}\right]^{\kappa^{+}}$.

Then $1 \Vdash_{\mathbb{P}(\bar{z})} \operatorname{No}\left(\sim_{\phi, \tilde{R}}\right)=\theta$.

Proof. Let $\widetilde{\mathcal{F}}_{\bar{z}}$ be a $\mathbb{P}(\bar{z})$-name for the set of all functions from $\kappa$ into 2, i.e., $\mathbf{1} \Vdash_{\mathbb{P}(\bar{z})} \widetilde{\mathcal{F}}_{\bar{z}}={ }^{\kappa} 2$. We prove that

$$
\mathbf{1} \Vdash_{\mathbb{P}(\bar{\mu})} \text { "for every } f \in{ }^{\kappa} 2 \text { there is } g \in \widetilde{\mathcal{F}}_{\bar{z}} \text { with } f \sim_{\phi, \tilde{R}} g \text { ". }
$$

This suffices since then

$$
\mathbf{1} \Vdash_{\mathbb{P}(\bar{\mu})} \theta \leq \operatorname{card}\left(\widetilde{\mathcal{F}}_{\bar{z}} / \sim_{\phi, \tilde{R}}\right) \leq \operatorname{card}\left({ }^{\kappa} 2 / \sim_{\phi, \tilde{R}}\right)=\operatorname{No}\left(\sim_{\phi, \tilde{R}}\right)=\theta,
$$

and by Lemma 4.2 , we can conclude

$$
\mathbf{1} \Vdash_{\mathbb{P}(\bar{z})} \operatorname{No}\left(\sim_{\phi, \tilde{R}}\right)=\operatorname{card}\left(\widetilde{\mathcal{F}}_{\bar{z}} / \sim_{\phi, \tilde{R}}\right)=\theta .
$$

Now assume, contrary to (A), that (4.1) holds and there are a condition $p$ in $\mathbb{P}(\bar{\mu})$ together with a $\mathbb{P}(\bar{\mu})$-name $\sigma$ for a function from $\kappa$ into 2 such that

$$
p \Vdash_{\mathbb{P}(\bar{\mu})} \text { "for all } g \in \widetilde{\mathcal{F}}_{\bar{z}}, \sigma \nsucc_{\phi, \tilde{R}} g \text { ". }
$$

Without loss of generality, the name $\sigma$ has cardinality $\kappa$. By Lemma 4.2, and since each cardinal in $\bar{\mu}$ is listed $\lambda$ times, we may choose $p$ and the name $\sigma$ so that the coordinates appearing in $\sigma$ add a tree with the same number of $\kappa$-branches as some coordinate in $\operatorname{dom}(\bar{z})$ does, i.e., for every $\xi \in \Delta_{1 s t}^{\sigma}$, there is $\zeta \in \operatorname{dom}(\bar{z})$ with $\mu_{\zeta}=\mu_{\xi}$. This property will be essential in the choice of automorphisms.

Our strategy will be as follows.

(i) We define a name $\sigma^{\prime}$ so that $\mathbf{1} \Vdash_{\mathbb{P}(\bar{\mu})} \sigma^{\prime} \in \widetilde{\mathcal{F}}_{\bar{z}}$. Hence, by applying (B), we get

$$
p \Vdash_{\mathbb{P}(\bar{\mu})} \sigma \chi_{\phi, \tilde{R}} \sigma^{\prime} .
$$

(ii) We define $\mathbb{P}(\bar{\mu})$-names $\left\langle\tau^{\gamma} \mid \gamma<\theta^{+}\right\rangle$for functions from $\kappa$ into 2 , and conditions $\left\langle q^{\gamma} \mid \gamma<\theta^{+}\right\rangle$in $\mathbb{P}(\bar{\mu})$. 
(iii) For every $\gamma<\gamma^{\prime}<\theta^{+}$we define a mapping $\varrho^{\gamma, \gamma^{\prime}}$ in $\operatorname{Mps}(\bar{\mu})$ such that $\varrho^{\gamma, \gamma^{\prime}}$ determines an automorphism $\widehat{\varrho}^{\gamma, \gamma^{\prime}}$ of $\mathbb{P}(\bar{\mu})$ with the following properties: $\widehat{\varrho}^{\gamma, \gamma^{\prime}}(\widetilde{R})=\widetilde{R}, \widehat{\varrho}^{\gamma, \gamma^{\prime}}(p)=q^{\gamma}, \varrho^{\gamma, \gamma^{\prime}}(\sigma)=\tau^{\gamma}$, and $\widehat{\varrho}^{\gamma, \gamma^{\prime}}\left(\sigma^{\prime}\right)=\tau^{\gamma^{\prime}}$. Hence it follows from (i) that

$$
q^{\gamma} \Vdash_{\mathbb{P}(\bar{\mu})} \tau^{\gamma} \chi_{\phi, \widetilde{R}} \tau^{\gamma^{\prime}}
$$

(iv) Finally, we fix a $\mathbb{P}(\bar{\mu})$-generic set $G$ over $V$ and, by applying a "standard density argument", we show that for some $B \in\left[\theta^{+}\right]^{\theta^{+}}$, all the conditions $q^{\gamma}, \gamma \in B$, are in the generic set $G$. It follows from (iii) that in $\mathrm{V}[G]$, $\operatorname{No}\left(\sim_{\phi, R}\right) \geq \theta^{+}$contrary to (4.1).

As can be guessed from the demands on the sequence $\bar{z}$, there are three different kinds of indices which we have to deal with:

$$
\Theta_{\leq}=\left\{\xi \in \Delta_{1 s t}^{\widetilde{R}} \mid \mu_{\xi} \leq \theta\right\}, \quad \Theta_{>}=\left\{\xi \in \Delta_{1 s t}^{\widetilde{R}} \mid \mu_{\xi}>\theta\right\}, \quad \Theta^{\prime}=\lambda \backslash \Delta_{1 s t}^{\widetilde{R}} .
$$

REMARK. Of course we would like to have $q^{\gamma}=\varrho^{\gamma, \gamma^{\prime}}(p)=p$ for every $\gamma<\gamma^{\prime}<\theta^{+}$. Unfortunately, that is not possible since it might be the case that for some $\xi \in \Theta_{>}, \Delta_{\xi}^{\sigma} \cap \Delta_{\xi}^{p} \not z_{\xi}$ (and we really need later the restriction $\left.\operatorname{card}\left(z_{\xi}\right)<\theta\right)$.

(i) We define the name $\sigma^{\prime}$ to be $\pi(\sigma)$ for a mapping $\pi$ in $\operatorname{Mps}(\bar{\mu})$ which satisfies the following conditions:

- $\operatorname{dom}(\pi)=\Delta^{\sigma}$;

- $\operatorname{ran}(\pi) \subseteq \Delta^{\bar{z}}$

- for every $\xi \in \operatorname{dom}\left(\pi_{1 s t}\right), \mu_{\left(\pi_{1 s t}(\xi)\right)}=\mu_{\xi}$;

- $\pi\left\lceil\Delta^{\widetilde{R}}\right.$ is identity (implying $\pi_{1 s t} \uparrow\left(\Theta_{\leq} \cup \Theta_{>}\right)$is identity);

- for every $\xi \in \operatorname{dom}\left(\pi_{1 s t}\right) \cap \Theta_{\leq}, \pi_{\xi}$ is identity;

- for every $\xi \in \operatorname{dom}\left(\pi_{1 s t}\right) \cap \Theta_{>}$and $\delta \in \operatorname{dom}\left(\pi_{\xi}\right) \backslash \Delta_{\xi}^{\widetilde{R}}, \pi_{\xi}(\delta) \notin \Delta_{\xi}^{p} \cup$ $\Delta_{\xi}^{\widetilde{R}} \cup \Delta_{\xi}^{\sigma} ;$

- for every $\xi \in \operatorname{dom}\left(\pi_{1 s t}\right) \cap \Theta^{\prime}, \pi_{1 s t}(\xi) \notin \operatorname{dom}(p) \cup \Delta_{1 s t}^{\widetilde{R}} \cup \Delta_{1 s t}^{\sigma}$ and $\pi_{\xi}$ is some injective function having range $z_{\xi}$.

It is possible to fulfill these conditions by the choice of $\sigma$, because of the cardinality demands on $\bar{z}$, and since $\Delta^{p} \cup \Delta^{\widetilde{R}} \cup \Delta^{\sigma}$ has cardinality $\kappa$. Since $\mathbf{1} \Vdash_{\mathbb{P}(\bar{\mu})} \sigma \in{ }^{\kappa} 2$ and $\pi$ can be extended so that the extension determines an automorphism of $\mathbb{P}(\bar{\mu})$, we have $\mathbf{1} \Vdash_{\mathbb{P}(\bar{\mu})} \sigma^{\prime} \in{ }^{\kappa} 2$. However, $\sigma^{\prime}$ is a $\mathbb{P}(\bar{z})$-name, so $\mathbf{1} \Vdash_{\mathbb{P}(\bar{\mu})} \sigma^{\prime} \in \widetilde{\mathcal{F}}_{\bar{z}}$ holds, too.

(ii) For every $\gamma<\theta^{+}$, we define a mapping $\pi^{\gamma} \in \operatorname{Mps}(\bar{\mu})$ so that the desired name $\tau^{\gamma}$ is $\pi^{\gamma}(\sigma)$ and the condition $q^{\gamma}$ is $\pi^{\gamma}(p)$. Since we do NOT demand that $\operatorname{ran}\left(\pi^{\gamma}\right) \subseteq \Delta^{\bar{z}}$, when $\gamma<\theta^{+}$, it is possible to choose $\pi^{\gamma}$ so that all the following demands are fulfilled:

- $\operatorname{dom}\left(\pi^{\gamma}\right)=\Delta^{\sigma} \cup \Delta^{p}$ 
- $\pi^{\gamma} \Delta^{\widetilde{R}}$ is identity;

- for every $\xi \in \operatorname{dom}\left(\pi_{1 s t}^{\gamma}\right), \mu_{\left(\pi_{1 s t}^{\gamma}(\xi)\right)}=\mu_{\xi}$;

- for every $\xi \in \operatorname{dom}\left(\pi_{1 s t}^{\gamma}\right) \cap\left(\Theta_{\leq} \cup \Theta^{\prime}\right), \pi_{\xi}^{\gamma}$ is identity;

- for all $\xi \in \operatorname{dom}\left(\pi_{1 s t}^{\gamma}\right) \cap \Theta_{>}$, the sets $\left(\Delta_{\xi}^{\widetilde{R}} \cup \Delta_{\xi}^{\sigma} \cup \Delta_{\xi}^{\sigma^{\prime}} \cup \Delta_{\xi}^{p}\right)$ and $\operatorname{ran}\left(\pi_{\xi}^{\gamma}\right) \backslash$ $\Delta_{\xi}^{\widetilde{R}}$, for all $\gamma<\theta^{+}$, are pairwise disjoint;

- the sets $\left(\Delta_{1 s t}^{\widetilde{R}} \cup \Delta_{1 s t}^{\sigma} \cup \Delta_{1 s t}^{\sigma^{\prime}} \cup \operatorname{dom}(p)\right)$ and $\operatorname{ran}\left(\pi_{1 s t}^{\gamma}\right) \backslash \Delta_{1 s t}^{\widetilde{R}}$, for all $\gamma<\theta^{+}$, are pairwise disjoint.

(iii) Fix indices $\gamma<\gamma^{\prime}<\theta^{+}$. Consider the set of pairs $\langle x, y\rangle$ such that

- $x \in \operatorname{dom}\left(\pi^{\gamma}\right)$ and $\pi^{\gamma}(x)=y$, or

- there is $z \in \operatorname{dom}(\pi)=\Delta^{\sigma}$ such that $\pi(z)=x$ and $\pi^{\gamma^{\prime}}(z)=y$.

Because of the conditions given above, we have

- for all $\xi \in \operatorname{dom}\left(\pi_{1 s t}^{\gamma}\right)=\operatorname{dom}\left(\pi_{1 s t}^{\gamma^{\prime}}\right), \pi_{1 s t}^{\gamma}(\xi)=\pi_{1 s t}^{\gamma^{\prime}}(\xi)$ iff $\pi_{1 s t}(\xi)=\xi$;

- for all $\langle\xi, \delta\rangle \in \operatorname{dom}\left(\pi^{\gamma}\right)=\operatorname{dom}\left(\pi^{\gamma^{\prime}}\right), \pi_{\xi}^{\gamma}(\delta)=\pi_{\xi}^{\gamma^{\prime}}(\delta)$ iff $\pi_{\xi}(\delta)=\delta$;

- for all $\xi \neq \zeta \in \operatorname{dom}\left(\pi_{1 s t}^{\gamma}\right), \pi_{\xi}^{\gamma}(\delta) \neq \pi_{\zeta}^{\gamma^{\prime}}(\varepsilon)$;

- for all $\langle\xi, \delta\rangle \neq\langle\xi, \varepsilon\rangle \in \operatorname{dom}\left(\pi^{\gamma}\right), \pi_{\xi}^{\gamma}(\delta) \neq \pi_{\xi}^{\gamma^{\prime}}(\varepsilon)$.

Hence the set of pairs we consider is the following well-defined injective function from $\operatorname{Mps}(\bar{\mu})$ :

$$
\eta=\pi^{\gamma} \cup\left(\left(\pi^{\gamma^{\prime}}\lceil\operatorname{dom}(\pi)) \circ(\pi)^{-1}\right) .\right.
$$

We let the mapping $\varrho^{\gamma, \gamma^{\prime}}$ be any extension of $\eta$ satisfying $\varrho^{\gamma, \gamma^{\prime}} \in \operatorname{Mps}(\bar{\mu})$, $\operatorname{dom}\left(\varrho_{1 s t}^{\gamma, \gamma^{\prime}}\right)=\lambda$, and for each $\xi<\lambda, \operatorname{dom}\left(\varrho_{\xi}^{\gamma, \gamma^{\prime}}\right)=\mu_{\xi}$. It follows that

- $\varrho^{\gamma, \gamma^{\prime}}(\widetilde{R})=\pi(\widetilde{R})=\pi^{\gamma}(\widetilde{R})=\pi^{\gamma^{\prime}}(\widetilde{R})=\widetilde{R}$;

- $\varrho^{\gamma, \gamma^{\prime}}(p)=\pi^{\gamma}(p)=q^{\gamma}$ (note that $\left.\operatorname{ran}(\pi) \cap\left(\Delta^{p} \backslash \Delta^{\widetilde{R}}\right)=\emptyset\right)$;

- $\varrho^{\gamma, \gamma^{\prime}}(\sigma)=\pi^{\gamma}(\sigma)=\tau^{\gamma}$ (note that $\left.\operatorname{ran}(\pi) \cap\left(\Delta^{\sigma} \backslash \Delta^{\widetilde{R}}\right)=\emptyset\right)$;

- $\varrho^{\gamma, \gamma^{\prime}}\left(\sigma^{\prime}\right)=\pi^{\gamma^{\prime}}\left(\pi^{-1}\left(\sigma^{\prime}\right)\right)=\pi^{\gamma^{\prime}}(\sigma)=\tau^{\gamma^{\prime}}$ (remember that $\pi(\sigma)=\sigma^{\prime}$ ).

(iv) Our demands on the mappings $\pi^{\gamma}, \gamma<\theta^{+}$, ensure that for each $\langle\xi, \delta\rangle \in \Delta^{p}$, if $\langle\xi, \delta\rangle \in \Delta^{\left(q^{\gamma}\right)}$ then $b_{\xi, \delta}^{\left(q^{\gamma}\right)}=b_{\xi, \delta}^{p}$. Therefore, $p$ and $q^{\gamma}$ are compatible conditions. Moreover, for every $\beta<\theta^{+}$, the set

$$
D_{\beta}=\left\{r \in \mathbb{P}(\bar{\mu}) \mid \text { for some } \gamma>\beta, r \leq q^{\gamma}\right\}
$$

is a dense set below the condition $p$ (which means that for every $s \leq p$ there is $r \leq s$ with $r \in D_{\beta}$ ). Since $p \in G, D_{\beta} \cap G$ is nonempty for every $\beta<\theta^{+}$. Consequently, the set $B=\left\{\gamma<\theta^{+} \mid q^{\gamma} \in G\right\}$ must be cofinal in $\theta^{+}$. So $B$ has cardinality $\theta^{+}$.

4.2. Isomorphism classes of names. First of all we fix $\bar{z}$ so that the subforcing $\mathbb{P}(\bar{z})$ of $\mathbb{P}(\bar{\mu})$ satisfies the assumptions of Lemma 4.3. Secondly 
we fix $\mathbb{P}(\bar{z})$-names $\left\langle\sigma_{\alpha} \mid \alpha<\theta\right\rangle$ for functions from $\kappa$ into 2 so that for all $\alpha \neq \beta<\theta$,

$$
1 \Vdash_{\mathbb{P}(\bar{z})} \sigma_{\alpha} \chi_{\phi, \tilde{R}} \sigma_{\beta} .
$$

Since $\mathbb{P}(\bar{z})$ has $\kappa^{+}$-c.c., we may assume that each of the names $\sigma_{\alpha}$ has cardinality $\kappa$.

Definition 4.4. For every $\alpha<\theta$ we fix an enumeration $\left\langle\left\langle\xi_{i}^{\alpha}, \delta_{i}^{\alpha}\right\rangle \mid i<\kappa\right\rangle$ of $\Delta^{\sigma_{\alpha}}$ without repetition. Names $\sigma_{\alpha}$ and $\sigma_{\beta}$ are said to be isomorphic, written $\sigma_{\alpha} \cong \sigma_{\beta}$, if the following conditions are met:

- for every $i<\kappa, \xi_{i}^{\alpha}=\xi_{i}^{\beta}$;

- for every $i<\kappa$ and $\zeta=\xi_{i}^{\alpha}=\xi_{i}^{\beta}$, if $\mu_{\zeta}>\theta$ then also $\delta_{i}^{\alpha}=\delta_{i}^{\beta}$;

- for all $\langle\zeta, \varepsilon\rangle \in \Delta^{\widetilde{R}}$ and $i\left\langle\kappa,\left\langle\xi_{i}^{\alpha}, \delta_{i}^{\alpha}\right\rangle=\langle\zeta, \varepsilon\rangle\right.$ iff $\left\langle\xi_{i}^{\beta}, \delta_{i}^{\beta}\right\rangle=\langle\zeta, \varepsilon\rangle$;

- $\pi\left(\sigma_{\alpha}\right)=\sigma_{\beta}$ when $\pi \in \operatorname{Mps}(\bar{z})$ is the mapping with $\operatorname{dom}(\pi)=\Delta^{\sigma_{\alpha}}$ and $\pi\left\langle\xi_{i}^{\alpha}, \delta_{i}^{\alpha}\right\rangle=\left\langle\xi_{i}^{\beta}, \delta_{i}^{\beta}\right\rangle$ for each $i<\kappa$.

For every $\alpha<\theta$ we denote the set $\left\{\beta<\theta \mid \sigma_{\beta} \cong \sigma_{\alpha}\right\}$ by $\Lambda^{\alpha}$. Now by the choice of $\mathbb{P}(\bar{z})$, and the assumptions $\kappa^{<\kappa}=\kappa$ and $2^{\kappa}=\kappa^{+}$, the number of nonisomorphic names in $\left\{\sigma_{\alpha} \mid \alpha<\theta\right\}$ is $\leq \kappa^{+}$, i.e., the cardinality of the family $\left\{\Lambda^{\alpha} \mid \alpha<\theta\right\}$ is at most $\kappa^{+}$.

Let $\Gamma$ be a subset of $\theta$ such that $\operatorname{card}(\Gamma) \leq \kappa^{+}$and $\left\{\sigma_{\alpha} \mid \alpha \in \Gamma\right\}$ is a set of representatives of the isomorphism classes. Since $\theta>\kappa^{+}$, the following equation holds:

$$
\theta=\bigcup_{\alpha \in \Gamma} \operatorname{card}\left(\Lambda^{\alpha}\right)
$$

Define the "set of all small cardinals" to be

$$
\operatorname{SC}(\widetilde{R})=\left\{\mu_{\xi} \mid \xi \in \Delta_{1 s t}^{\widetilde{R}} \text { and } \mu_{\xi} \leq \theta\right\} .
$$

Note that this set might be empty. Anyway, we know that

$$
\theta \geq \max \left\{\kappa^{++}, \sup \operatorname{SC}(\widetilde{R})\right\} .
$$

To prove that $\theta$ is a cardinal in $\Omega_{\bar{\mu}}$ we shall show that for every $\alpha \in \Gamma$, the cardinality of $\Lambda^{\alpha}$ is strictly smaller than the lower bound given in (4.4) above, or otherwise, we can find a subset $I^{\alpha}$ of $\kappa$ so that $\operatorname{card}\left(\Lambda^{\alpha}\right)$ has one of the following forms: either $\operatorname{card}\left(I^{\alpha}\right)<\kappa$ and

$$
\operatorname{card}\left(\Lambda^{\alpha}\right) \in\left\{\bigcup_{i \in I^{\alpha}} \mu_{\xi_{i}^{\alpha}}\right\} \cup\left\{\prod_{i \in I^{\alpha}} \mu_{\xi_{i}^{\alpha}}\right\} \quad \text { (Subsection 4.3), }
$$

or else, $\operatorname{card}\left(I^{\alpha}\right)=\kappa$ and

$$
\operatorname{card}\left(\Lambda^{\alpha}\right)=\bigcup_{K \in\left[I^{\alpha}\right]<\kappa} \operatorname{card}\left(\prod_{i \in K} \mu_{\xi_{i}^{\alpha}}\right) \quad \text { (Subsection 4.4). }
$$


This suffices, since we may ensure that for every $\alpha \in \Gamma$ and for each $i \in I^{\alpha}$, the cardinal $\mu_{\xi_{i}^{\alpha}}$ is in $\mathrm{SC}(\widetilde{R})$. This means that only those small cardinals are used whose coordinates appear in the name $\widetilde{R}$. It follows that there occurs at most $\kappa$ different cardinals in the union (4.3). Hence, for some sequence $\left\langle X_{k} \mid k<\kappa\right\rangle$ of sets in $[\mathrm{SC}(\widetilde{R})]^{<\kappa}$,

$$
\theta=\bigcup_{k<\kappa} \operatorname{card}\left(\prod_{\mu \in X_{k}} \mu\right) \in \Omega_{\bar{\mu}}
$$

REMARK. From our assumption that for every $\chi \in \Omega_{\bar{\mu}} \backslash \kappa^{++}$and $\gamma<$ $\kappa$, the inequality $\chi^{\gamma} \leq \chi^{+}$holds, it follows that $\theta$ is either $\sup \operatorname{SC}(\widetilde{R})$ or $\operatorname{card}\left(\prod_{\mu \in X} \mu\right)$ for some subset $X$ of $\operatorname{SC}(\widetilde{R})$ with $\operatorname{card}(X)<\kappa$.

4.3. Case 1: The parameter depends on less than $\kappa$ coordinates. For the rest of the proof, let $\alpha^{*}$ be a fixed ordinal so that the number of names in $\left\{\sigma_{\beta} \mid \beta<\theta\right\}$ which are isomorphic to the representative $\sigma_{\alpha^{*}}$ is greater than or equal to the lower bound given in (4.4), i.e., $\alpha^{*} \in \Gamma$ and $\operatorname{card}\left(\Lambda^{\alpha^{*}}\right)$ is at least $\max \left\{\kappa^{++}, \sup \operatorname{SC}(\widetilde{R})\right\}$.

To simplify our notation, let $\bar{\xi}^{*}=\left\langle\xi_{i}^{*} \mid i<\kappa\right\rangle$ and $\bar{\delta}^{*}=\left\langle\delta_{i}^{*} \mid i<\kappa\right\rangle$ denote the sequences $\bar{\xi}^{\alpha^{*}}$ and $\bar{\delta}^{\alpha^{*}}$ respectively, and abbreviate $\Lambda^{\alpha^{*}}$ by $\Lambda^{*}$.

Define the set of "critical indices of the isomorphism class of $\sigma_{\alpha^{*}}$ " to be

$$
J^{*}=\left\{i<\kappa \mid \mu_{\xi_{i}^{*}} \leq \theta \text { and }\left\langle\xi_{i}^{*}, \delta_{i}^{*}\right\rangle \notin \Delta^{\widetilde{R}}\right\} .
$$

Note that for every $\alpha \in \Lambda^{*}$, we have $\bar{\xi}^{\alpha}=\bar{\xi}^{*}$ and $\bar{\delta}^{\alpha} \uparrow\left(\kappa \backslash J^{*}\right)=\bar{\delta}^{*} \uparrow\left(\kappa \backslash J^{*}\right)$. Note also that by the choice of $\mathbb{P}(\bar{z}), J^{*} \subseteq\left\{i<\kappa \mid z_{\xi_{i}^{*}}=\mu_{\xi_{i}^{*}}\right\} \subseteq\{i<\kappa \mid$ $\left.\xi_{i}^{*} \in \Delta_{1 s t}^{\widetilde{R}}\right\}$. Thus also $\left\{\mu_{\xi_{i}^{*}} \mid i \in J^{*}\right\} \subseteq \operatorname{SC}(\widetilde{R})$.

The set $J^{*}$ must be nonempty, since otherwise there are $\alpha \neq \beta$ in $\Lambda^{*}$ such that $\sigma_{\alpha}$ is the same name as $\sigma_{\beta}$, contrary to the choice that $\sigma_{\alpha}$ and $\sigma_{\beta}$ are names for nonequivalent functions (see (4.2)). For a similar reason we have $\operatorname{card}\left(\prod_{i \in J^{*}} \mu_{\xi_{i}^{*}}\right) \geq \operatorname{card}\left(\Lambda^{*}\right)$.

Now suppose that a subset $K$ of $J^{*}$ having cardinality $<\kappa$ satisfies

$$
\operatorname{card}\left(\prod_{i \in K} \mu_{\xi_{i}^{*}}\right) \geq \operatorname{card}\left(\Lambda^{*}\right) .
$$

If $\operatorname{card}\left(\Lambda^{*}\right)=\operatorname{card}\left(\prod_{i \in K} \mu_{\xi_{i}^{*}}\right)$ we can define $I^{\alpha^{*}}$ to be $K$. Otherwise, our assumption on the cardinal arithmetic gives

$$
\operatorname{card}\left(\prod_{i \in K} \mu_{\xi_{i}^{*}}\right)=\left(\bigcup_{i \in K} \mu_{\xi_{i}^{*}}\right)^{+}>\operatorname{card}\left(\Lambda^{*}\right) .
$$

By the choice of $\alpha^{*}, \operatorname{card}\left(\Lambda^{*}\right) \geq \sup \operatorname{SC}(\widetilde{R}) \geq \bigcup_{i \in K} \mu_{\xi_{i}^{*}}$. Hence $\operatorname{card}\left(\Lambda^{*}\right)=$ $\bigcup_{i \in K} \mu_{\xi_{i}^{*}}$ and again we can choose $I^{\alpha^{*}}$ to be $K$.

It follows that when $\operatorname{card}\left(J^{*}\right)<\kappa$ we can find $I^{\alpha^{*}}$ satisfying (4.5). 


\subsection{Case 2: The parameter depends on $\kappa$ coordinates}

REMARK. If $\bar{\mu}$ is such that each $\mu_{\xi}$ is $\kappa^{+}$or $\lambda$, we have proved so far that $\theta$ must be either $\leq \kappa^{+}$or $\theta=\lambda$.

For the rest of the proof we assume that the set $J^{*}$ given in (4.7) has cardinality $\kappa$ and for every $K \in\left[J^{*}\right]^{<\kappa}, \operatorname{card}\left(\prod_{i \in K} \mu_{\xi_{i}^{*}}\right)<\operatorname{card}\left(\Lambda^{*}\right)$. So $\chi^{*} \leq \operatorname{card}\left(\Lambda^{*}\right)$, where

$$
\chi^{*}=\bigcup_{K \in\left[J^{*}\right]<\kappa} \operatorname{card}\left(\prod_{i \in K} \mu_{\xi_{i}^{*}}\right) .
$$

We prove that also card $\left(\Lambda^{*}\right) \leq \chi^{*}$, and thus, (4.6) is fulfilled. The intuition behind the forthcoming "technical tools" is simple and explained at the beginning of this section (right before Subsection 4.1). As mentioned in that introduction, we need to define "coherent names". But it is easier to look at a "list of indices" than the real names, and define that a "neat pair of lists" produces "coherent names" (Definition 4.5). The fact that "coherent names can be copied by automorphisms of $\mathbb{P}(\bar{z})$ " is presented in Lemma 4.6.

Definition 4.5. Define $\mathcal{E}^{*}$ to be the set of all sequences $\bar{\varepsilon}=\left\langle\varepsilon_{i} \mid i<\kappa\right\rangle$ such that

- for each $i \in J^{*}, \varepsilon_{i} \in \mu_{\xi_{i}^{*}} \backslash \Delta_{\xi_{i}^{*}}^{\widetilde{R}}$;

- for each $i \in \kappa \backslash J^{*}, \varepsilon_{i}=\delta_{i}^{*}$;

- for every $i<j<\kappa,\left\langle\xi_{i}^{*}, \varepsilon_{i}\right\rangle \neq\left\langle\xi_{j}^{*}, \varepsilon_{j}\right\rangle$.

Again, to simplify our notation, we write $\pi(\bar{\delta})$ for the sequence $\left\langle\pi_{\xi_{i}^{*}}\left(\delta_{i}\right) \mid i<\kappa\right\rangle$ when $\bar{\delta}$ is in $\mathcal{E}^{*}$ and $\pi$ in $\operatorname{Mps}(\bar{z})$ satisfies $\left\{\left\langle\xi_{i}^{*}, \delta_{i}\right\rangle \mid i<\kappa\right\} \subseteq \operatorname{dom}(\pi)$.

Every sequence $\bar{\varepsilon}$ in $\mathcal{E}^{*}$ determines a $\mathbb{P}(\bar{z})$-name $\tau_{\bar{\varepsilon}}$ for a function from $\kappa$ into 2. Namely, we define $\tau_{\bar{\varepsilon}}$ to be the name $\pi\left(\sigma_{\alpha^{*}}\right)$ where $\pi$ is the mapping in $\operatorname{Mps}(\bar{z})$ satisfying $\operatorname{dom}(\pi)=\left\{\left\langle\xi_{i}^{*}, \delta_{i}^{*}\right\rangle \mid i<\kappa\right\}$ and $\pi\left(\bar{\delta}^{*}\right)=\bar{\varepsilon}$.

A pair $\langle\bar{\delta}, \bar{\varepsilon}\rangle$ of sequences in $\mathcal{E}^{*}$ is called a neat pair if for all $i<j<\kappa$, $\left\langle\xi_{i}^{*}, \delta_{i}\right\rangle \neq\left\langle\xi_{j}^{*}, \varepsilon_{j}\right\rangle$.

Denote by $A(\bar{\delta}, \bar{\varepsilon})$ the set $\left\{i \in J^{*} \mid \delta_{i}=\varepsilon_{i}\right\}$ for $\bar{\delta}, \bar{\varepsilon} \in \mathcal{E}^{*}$.

The sequence $\bar{\delta}^{\alpha}$ is in $\mathcal{E}^{*}$ when $\alpha \in \Lambda^{*}$. Also $\tau_{\bar{\delta}^{\alpha}}$ is the name $\sigma_{\alpha}$ for every $\alpha \in \Lambda^{*}$. In fact, $\left\{\tau_{\bar{\varepsilon}} \mid \bar{\varepsilon} \in \mathcal{E}^{*}\right\}$ is the collection of all the $\mathbb{P}(\bar{z})$-names which are "isomorphic" to the fixed representative $\sigma_{\alpha^{*}}$.

Lemma 4.6. Suppose $\bar{\delta}^{1}, \bar{\delta}^{2}, \bar{\varepsilon}^{1}, \bar{\varepsilon}^{2} \in \mathcal{E}^{*}$ are such that both $\left\langle\bar{\delta}^{1}, \bar{\varepsilon}^{1}\right\rangle$ and $\left\langle\bar{\delta}^{2}, \bar{\varepsilon}^{2}\right\rangle$ are neat, and moreover $A\left(\bar{\delta}^{1}, \bar{\varepsilon}^{-1}\right)=A\left(\bar{\delta}^{2}, \bar{\varepsilon}^{2}\right)$. Then there is an automorphism $\widehat{\pi}$ of $\mathbb{P}(\bar{z})$ such that $\widehat{\pi}(\widetilde{R})=\widetilde{R}, \widehat{\pi}\left(\tau_{\bar{\delta}^{1}}\right)=\tau_{\bar{\delta}^{2}}$ and $\widehat{\pi}\left(\tau_{\bar{\varepsilon}^{1}}\right)=\tau_{\bar{\varepsilon}^{2}}$. Hence for every $p \in \mathbb{P}(\bar{z})$,

$$
p \Vdash_{\mathbb{P}(\bar{z})} \tau_{\bar{\delta}^{1}} \sim_{\phi, \tilde{R}} \tau_{\bar{\varepsilon}^{1}} \quad \text { iff } \quad \widehat{\pi}(p) \Vdash_{\mathbb{P}(\bar{z})} \tau_{\bar{\delta}^{2}} \sim_{\phi, \widetilde{R}} \tau_{\bar{\varepsilon}^{2}} .
$$

Proof. There is a mapping $\pi$ in $\operatorname{Mps}(\bar{z})$ such that $\pi\left(\bar{\delta}^{1}\right)=\bar{\delta}^{2}$ and $\pi\left(\bar{\varepsilon}^{1}\right)=\bar{\varepsilon}^{2}$, because the sequences in $\mathcal{E}^{*}$ are without repetition, both pairs 
are neat, and $A\left(\bar{\delta}^{1}, \bar{\varepsilon}^{1}\right)=A\left(\bar{\delta}^{2}, \bar{\varepsilon}^{2}\right)$. Furthermore, $\pi$ can be chosen so that $\pi \uparrow \Delta^{\widetilde{R}}$ is identity and each $\pi_{\xi_{i}^{*}}$ is a permutation of $z_{\xi_{i}^{*}}$. Hence $\pi$ determines an automorphism as required.

For technical reasons we define

$\mathcal{A}^{*}=\left\{I \subseteq \kappa \mid\right.$ there are $\alpha \neq \beta \in \Lambda^{*}$ such that $\left\langle\bar{\delta}^{\alpha}, \bar{\delta}^{\beta}\right\rangle$ is neat and $\left.I \subseteq A\left(\bar{\delta}^{\alpha}, \bar{\delta}^{\beta}\right)\right\}$.

The next lemma explains why we closed the set $\mathcal{A}^{*}$ under subsets: all the names $\sigma_{\alpha}, \alpha \in \Lambda^{*}$, are forced to be nonequivalent, and moreover, all those names are forced to be nonequivalent which are determined by a neat pair of sequences agreeing in a smaller set than some pair of fixed sequences $\bar{\delta}^{\alpha}$, $\alpha \in \Lambda^{*}$.

Lemma 4.7. For all $\bar{\delta}, \bar{\varepsilon} \in \mathcal{E}^{*}$, if $\langle\bar{\delta}, \bar{\varepsilon}\rangle$ is neat and $A(\bar{\delta}, \bar{\varepsilon})$ is in $\mathcal{A}^{*}$, then $\mathbf{1} \Vdash_{\mathbb{P}(\bar{z})} \tau_{\bar{\delta}} \chi_{\phi, \widetilde{R}} \tau_{\bar{\varepsilon}}$.

Proof. First we fix $\alpha \neq \beta \in \Lambda^{*}$ and $I$ such that $\left\langle\bar{\delta}^{\alpha}, \bar{\delta}^{\beta}\right\rangle$ is neat and $I=A(\bar{\delta}, \bar{\varepsilon}) \subseteq A\left(\bar{\delta}^{\alpha}, \bar{\delta}^{\beta}\right)$. Let $\bar{\delta}^{\prime}$ be a sequence in $\mathcal{E}^{*}$ such that $\bar{\delta}^{\prime}\left\lceil I=\bar{\delta}^{\alpha}\lceil I\right.$ and for all $i \in J^{*} \backslash I, \delta_{i}^{\prime} \notin\left\{\delta_{j}^{\alpha} \mid j<\kappa\right\}$. Then the pair $\left\langle\bar{\delta}^{\prime}, \bar{\delta}^{\alpha}\right\rangle$ is neat and $A\left(\bar{\delta}^{\prime}, \bar{\delta}^{\alpha}\right)=I$. We want to show that $\mathbf{1} \Vdash_{\mathbb{P}(\bar{z})} \tau_{\bar{\delta}^{\prime}} \chi_{\phi, \tilde{R}} \tau_{\bar{\delta}^{\alpha}}$, because then it follows from Lemma 4.6 that $\mathbf{1} \Vdash_{\mathbb{P}(\bar{z})} \tau_{\bar{\delta}} \chi_{\phi, \tilde{R}} \tau_{\bar{\varepsilon}}$.

Suppose, contrary to this claim, that $p \in \mathbb{P}(\bar{z})$ satisfies

$$
p \Vdash_{\mathbb{P}(\bar{z})} \tau_{\bar{\delta}^{\prime}} \sim_{\phi, \tilde{R}} \tau_{\bar{\delta}^{\alpha}} .
$$

Let $J$ denote the set $A\left(\bar{\delta}^{\alpha}, \bar{\delta}^{\beta}\right)$ and choose a sequence $\bar{\varepsilon}^{\prime}$ from $\mathcal{E}^{*}$ so that $\bar{\delta}^{\beta}\left\lceil J=\bar{\varepsilon}^{\prime} \nmid J\right.$ and for all $i \in J^{*} \backslash J$,

$$
\varepsilon_{i}^{\prime} \notin \Delta_{\xi_{i}^{*}}^{p} \cup\left\{\delta_{j}^{\prime} \mid j<\kappa\right\} \cup\left\{\delta_{j}^{\alpha} \mid j<\kappa\right\} .
$$

Then the pair $\left\langle\bar{\varepsilon}^{\prime}, \bar{\delta}^{\alpha}\right\rangle$ is neat and $A\left(\bar{\varepsilon}^{\prime}, \bar{\delta}^{\alpha}\right)=J$. By the choice of the names $\sigma_{\alpha}$ and $\sigma_{\beta}$, i.e., by $(4.2), \mathbf{1} \Vdash_{\mathbb{P}(\bar{z})} \sigma_{\alpha} \chi_{\phi, R} \sigma_{\beta}$. Once more, it follows from Lemma 4.6 that

$$
\mathbf{1} \Vdash_{\mathbb{P}(\bar{z})} \sigma_{\alpha}=\tau_{\bar{\delta}^{\alpha}} \chi_{\phi, R} \tau_{\bar{\varepsilon}^{\prime}} .
$$

Choose $\pi$ from $\operatorname{Mps}(\bar{z})$ so that $\pi(\widetilde{R})=\widetilde{R}, \pi\left(\bar{\delta}^{\prime}\right)=\bar{\delta}^{\prime}, \pi\left(\bar{\delta}^{\alpha}\right)=\bar{\varepsilon}^{\prime}$, $\pi \uparrow\left(\pi\left[\Delta^{p}\right] \cap \Delta^{p}\right)$ is identity, and $\pi$ determines an automorphism $\widehat{\pi}$ of $\mathbb{P}(\bar{z})$. This is possible by the choice of the sequence $\bar{\varepsilon}^{\prime}$. Since $A\left(\bar{\delta}^{\prime}, \bar{\varepsilon}^{\prime}\right)=A\left(\bar{\delta}^{\prime}, \bar{\delta}^{\alpha}\right)$ and $\left\langle\bar{\delta}^{\prime}, \bar{\varepsilon}^{\prime}\right\rangle$ is a neat pair, it follows from Lemma 4.6 that

$$
\widehat{\pi}(p) \Vdash_{\mathbb{P}(\bar{z})} \tau_{\bar{\delta}^{\prime}} \sim_{\phi, \tilde{R}} \tau_{\bar{\varepsilon}^{\prime}}
$$

Now there is $q \in \mathbb{P}(\bar{z})$ satisfying $q \leq p$ and $q \leq \widehat{\pi}(p)$. Since $\sim_{\phi, \tilde{R}}$ is a name for an equivalence relation, $q \Vdash_{\mathbb{P}(\bar{z})} \tau_{\bar{\delta}^{\alpha}} \sim_{\phi, \tilde{R}} \tau_{\bar{\varepsilon}^{\prime}}$, a contradiction.

Next we want to show that there is always a small set of indices outside of $\mathcal{A}^{*}$. 
Lemma 4.8. When $J^{*}$ has cardinality $\kappa$ there are $p \in \mathbb{P}(\bar{z})$ and a neat $\operatorname{pair}\langle\bar{\delta}, \bar{\varepsilon}\rangle$ of sequences in $\mathcal{E}^{*}$ satisfying

$$
A(\bar{\delta}, \bar{\varepsilon}) \in\left[J^{*}\right]^{<\kappa} \quad \text { and } \quad p \Vdash_{\mathbb{P}(\bar{z})} \tau_{\bar{\delta}} \sim_{\phi, \tilde{R}} \tau_{\bar{\varepsilon}} .
$$

Proof. First of all, for every $i \in J^{*}$ and $\eta \in{ }^{i} 2$ we fix an ordinal $\beta_{\eta}$ from $\mu_{\xi_{i}^{*}} \backslash \Delta_{\xi_{i}^{*}}^{\widetilde{R}}$ so that for all $i, j \in J^{*}, \eta \in{ }^{i} 2$, and $\nu \in{ }^{j} 2, \beta_{\eta}=\beta_{\nu}$ iff $i=j$ and $\eta=\nu$. Fix also a coordinate $\zeta<\lambda$ so that $\mu_{\zeta}>\theta$ and $\zeta \notin Z$ ( $\zeta$ is outside of $\mathbb{P}(\bar{z}))$. Suppose $G$ is a $P_{\mu_{\zeta}}$-generic set over V. For any function $u \in\left({ }^{\kappa} 2\right)^{\mathrm{V}[G]}$, let $\bar{\delta}^{u}$ denote the following sequence: $\bar{\delta}^{u}=\left\langle\delta_{i}^{u} \mid i<\kappa\right\rangle$, $\delta_{i}^{u}=\beta_{u \uparrow i}$ if $i \in J^{*}$, and $\delta_{i}^{u}=\delta_{i}^{*}$ otherwise. Then each of the sequences $\bar{\delta}^{u}$ is in $\left(\mathcal{E}^{*}\right)^{\mathrm{V}[G]}$. Moreover, $\left\langle\bar{\delta}^{u}, \bar{\delta}^{v}\right\rangle$ is a neat pair for all $u$ and $v$ in $\left({ }^{\kappa} 2\right)^{\mathrm{V}[G]}$.

Let $H$ be a $\mathbb{P}(\bar{z})$-generic set over $\mathrm{V}[G]$. In $\mathrm{V}[G]$, there are at least $\mu_{\zeta}$ different functions from $\kappa$ into 2. By assumption (4.1) and Lemma 4.2, there are only $\theta$ equivalence classes of $\sim_{\phi, R}$ in $\mathrm{V}[G][H]$. It follows that for some $p \in H$ and $u \neq v \in\left({ }^{\kappa} 2\right)^{\mathrm{V}[G]}$ the following holds in $\mathrm{V}[G]$ :

$$
p \Vdash_{\mathbb{P}(\bar{z})} \tau_{\bar{\delta}^{u}} \sim_{\phi, \widetilde{R}} \tau_{\bar{\delta}^{v}} .
$$

By the definition of the ordinals $\beta_{\nu}$, we have $A\left(\bar{\delta}^{u}, \bar{\delta}^{v}\right)=\left\{i \in J^{*} \mid u \nmid i=\right.$ $v\lceil i\} \in\left[J^{*}\right]^{<\kappa}$, and hence $A\left(\bar{\delta}^{u}, \bar{\delta}^{v}\right)$ is in V.

Now, in $\mathrm{V}$, we can fix a neat pair $\left\langle\bar{\varepsilon}^{1}, \bar{\varepsilon}^{2}\right\rangle$ of sequences in $\mathcal{E}^{*}$ such that $A\left(\bar{\varepsilon}^{1}, \bar{\varepsilon}^{2}\right)=A\left(\bar{\delta}^{u}, \bar{\delta}^{v}\right)$. Let $\pi \in(\operatorname{Mps}(\bar{z}))^{\mathrm{V}[G]}$ determine, in $\mathrm{V}[G]$, an automorphism $\widehat{\pi}$ of $\mathbb{P}(\bar{z})$ satisfying $\widehat{\pi}(\widetilde{R})=\widetilde{R}, \widehat{\pi}\left(\bar{\delta}^{u}\right)=\bar{\varepsilon}^{1}$, and $\widehat{\pi}\left(\bar{\delta}^{v}\right)=\bar{\varepsilon}^{2}$. For such a $\pi$ in $\mathrm{V}[G]$, we have $\widehat{\pi}(p) \Vdash_{\mathbb{P}(\bar{z})} \tau_{\bar{\varepsilon}^{1}} \sim_{\phi, \tilde{R}} \tau_{\bar{\varepsilon}^{2}}$. Note that the condition $q=\widehat{\pi}(p)$ is in $\mathrm{V}$. From the equivalence of the forcings $P_{\mu_{\zeta}} \times \mathbb{P}(\bar{z})$ and $\mathbb{P}(\bar{z}) \times P_{\mu_{\zeta}}$, together with Lemma 4.2, it follows that already in $\mathrm{V}, q \Vdash_{\mathbb{P}(\bar{z})} \tau_{\bar{\varepsilon}^{1}} \sim_{\phi, \tilde{R}^{2}} \tau_{\bar{\varepsilon}^{2}}$.

Finally, we claim that $\operatorname{card}\left(\Lambda^{*}\right)=\chi^{*}$, and thus we can satisfy (4.6). Suppose, contrary to this claim, that $\operatorname{card}\left(\Lambda^{*}\right)>\chi^{*}$. In the lemma below, we show that then all the subsets of $J^{*}$ of cardinality $<\kappa$ are in $\mathcal{A}^{*}$. It follows from Lemma 4.7 that for all $\bar{\delta}, \bar{\varepsilon} \in \mathcal{E}^{*}$, if $\langle\bar{\delta}, \bar{\varepsilon}\rangle$ is neat and $A(\bar{\delta}, \bar{\varepsilon})$ is of cardinality $<\kappa$, then $1 \Vdash_{\mathbb{P}(\bar{z})} \tau_{\bar{\delta}} \chi_{\phi, \tilde{R}} \tau_{\bar{\varepsilon}}$. By Lemma 4.8 , this leads to a contradiction. So it remains to prove the following last lemma.

Lemma 4.9. If $\operatorname{card}\left(\Lambda^{*}\right)>\chi^{*}$ then $\left[J^{*}\right]^{<\kappa} \subseteq \mathcal{A}^{*}$.

Proof. Fix a set $K$ from $\left[J^{*}\right]^{<\kappa}$. Since we have $\operatorname{card}\left(\Lambda^{*}\right)>\chi^{*} \geq$ $\operatorname{card}\left(\prod_{i \in K} \mu_{\xi_{i}^{*}}\right) \geq 2^{\kappa}$, there is $X_{1} \subseteq \Lambda^{*}$ of cardinality $\left(2^{\kappa}\right)^{+}$such that for all $\alpha \neq \beta \in X_{1}, K \subseteq A\left(\bar{\delta}^{\alpha}, \bar{\delta}^{\beta}\right)$. By the $\Delta$-lemma one can find $X_{2} \in\left[X_{1}\right]^{\left(2^{\kappa}\right)^{+}}$ such that for all $\alpha \neq \beta \in X_{2}$, the intersection $\left\{\delta_{i}^{\alpha} \mid i<\kappa\right\} \cap\left\{\delta_{i}^{\beta} \mid i<\kappa\right\}$ is some fixed set $\Xi$. There are also $I \subseteq \kappa$ and $X_{3} \in\left[X_{2}\right]^{\left(2^{\kappa}\right)^{+}}$such that for all $\alpha \in X_{3},\left\{i<\kappa \mid \delta_{i}^{\alpha} \in \Xi\right\}=I$. Hence there is $\alpha \neq \beta \in X_{3}$ with $\bar{\delta}^{\alpha}\left\lceil I=\bar{\delta}^{\beta}\lceil I\right.$ and $\left\{\delta_{i}^{\alpha} \mid i \in \kappa \backslash I\right\} \cap\left\{\delta_{i}^{\beta} \mid i \in \kappa \backslash I\right\}=\emptyset$, i.e., $\left\langle\bar{\delta}^{\alpha}, \bar{\delta}^{\beta}\right\rangle$ forms a neat pair with $K \subseteq I=A\left(\bar{\delta}^{\alpha}, \bar{\delta}^{\beta}\right)$. 
5. Remarks. The following facts are also useful to know, when applying the theorem proved. Write $\operatorname{Fn}(\kappa, 2, \kappa)$ for the ordinary Cohen forcing which adds a generic subset of $\kappa$, i.e., the forcing $\{\eta \mid \eta$ is a partial function from $\kappa$ into 2 and $\operatorname{card}(\eta)<\kappa\}$ ordered by reverse inclusion.

FACT 5.1. (a) There is a dense subset $Q \subseteq \operatorname{Fn}(\kappa, 2, \kappa)$ and a dense embedding of $Q$ into $P_{\kappa}$ (where $P_{\kappa}$ is the forcing adding a tree with $\kappa$ branches through it, see Definition 2.1).

(b) Every subforcing $\mathbb{P}(\bar{z})$ of $\mathbb{P}(\bar{\mu})$ is equivalent to $\operatorname{Fn}(\kappa, 2, \kappa)$ provided that the length of $\bar{z}$ is at most $\kappa$ and each $z_{\xi}$ has cardinality $\kappa$.

(c) The forcing $\mathbb{P}(\bar{\mu})$ is locally $\kappa$ Cohen, i.e., every subset $Q$ of $\mathbb{P}(\bar{\mu})$ of size $\leq \kappa$ is included in a complete subforcing $Q^{\prime}$ of $\mathbb{P}(\bar{\mu})$ so that $Q^{\prime}$ is equivalent to $\operatorname{Fn}(\kappa, 2, \kappa)$.

(d) Assume that $\kappa$ is a weakly compact cardinal, and $\mathrm{V}$ is such that $\kappa$ remains weakly compact after forcing with $\operatorname{Fn}(\kappa, 2, \kappa)$. Then every locally $\kappa$ Cohen forcing preserves weak compactness of $\kappa$.

Note that if $\kappa$ is a weakly compact cardinal then, using upward Easton forcing, it is possible to have a generic extension $\mathrm{V}[H]$ such that $\kappa$ is weakly compact in $\mathrm{V}[H]$ and $\kappa$ remains weakly compact in all extensions $\mathrm{V}[H][G]$, where $G$ is $\operatorname{Fn}(\kappa, 2, \kappa)$-generic over $\mathrm{V}[H]$ (an unpublished result by Silver). These facts are applied in [SV].

\section{References}

[Jec71] T. J. Jech, Trees, J. Symbolic Logic 36 (1971), 1-14.

[Jec97] —, Set Theory, 2nd ed., Springer, Berlin, 1997.

[Shea] S. Shelah, On nice equivalence relations on $2^{\lambda}$, Arch. Math. Logic, to appear.

[Sheb] -, Strong dichotomy of cardinality, Results Math. 39 (2001), 131-154.

[SV] S. Shelah and P. Väisänen, The number of $L_{\infty \kappa}$-equivalent nonisomorphic models for $\kappa$ weakly compact, Fund. Math., to appear.

Institute of Mathematics

The Hebrew University

Jerusalem, Israel

E-mail: shelah@math.huji.ac.il

Department of Mathematics

Rutgers University

New Brunswick, NJ 08903, U.S.A.

E-mail: shelah@math.rutgers.edu
Department of Mathematics

P.O. Box 4

00014 University of Helsinki, Finland

E-mail: pauli.vaisanen@helsinki.fi 